\title{
Dynamic Reference Point-Oriented Consensus Mechanism in Linguistic Distribution Group Decision Making Restricted by Quantum Integration of Information
}

\author{
Xiao $\operatorname{Tan}^{1} \cdot$ Jianjun Zhu ${ }^{1} \oplus \cdot$ Tong $\mathrm{Wu}^{1}$
}

Accepted: 27 January 2022 / Published online: 24 February 2022

(c) The Author(s), under exclusive licence to Springer Nature B.V. 2022

\begin{abstract}
We present a consensus improvement mechanism based on prospect theory and quantum probability theory (QPT) that enables the manifestation of irrational and uncertain behaviors of decision makers (DMs) in linguistic distribution group decision making. In this framework, the DMs pursue the possibility of working with different partial agreements on prospect values. Considering that the reference information should be comprehensive and accurate as it guides information modification and affects consensus efficiency, objective and subjective information is integrated to obtain the information. Several studies have verified that the interference effect will occur when the brain beliefs flow towards the different decision classification paths. To address this problem, QPT is introduced into the information integration and the optimized value of the interference term can be acquired by the designed multi-objective programming model based on the maximum individual utility. Finally, as the reference point changes during the preference adjustment process, a dynamic reference point-oriented consensus model is constructed to obtain the optimized modification. A case study is performed on the emergency plan for the selection of designated hospitals, and comparative analyses are performed to demonstrate the feasibility and advantages of the proposed model. Several important insights are offered to simulate the most likely possibility of consciousness flowing into different decision classifications for DMs and moderators.
\end{abstract}

Keywords Group decision and negotiation · Prospect theory · Quantum probability theory $\cdot$ Linguistic distribution assessment $\cdot$ Goal programming model $\cdot$ Reference point rules

Jianjun Zhu

zhujianjun@nuaa.edu.cn

Extended author information available on the last page of the article 


\section{Introduction}

Group decision making (GDM) aims at the optimal decision being made among multiple alternative options; communication, negotiation, and modification are necessary when an unacceptable disagreement arises (Labella et al. 2018; Zhang et al. 2019; Rodríguez et al. 2018). As opposed to the impractical "hard" consensus (Bezdek et al. 1978), the "soft" consensus has been widely explored, in which a consensus threshold is preset to measure whether or not the consensus is reached (Labella et al. 2020; Zhang et al. 2018; Wu et al. 2020; Xu et al. 2021). The key issue of the consensus reaching process (CRP) is to design an effective feedback mechanism, including the identification of unacceptable decision makers (DMs) (those who contribute less to the group opinion), the determination of reference information (for the identified DMs), and the modification rule (for the identified DMs). The existing literature provides series of consensus models; however, the common point that has not attracted sufficient attention is the psychological behavior of DMs showing in the CRP. Human rationality generally lies between complete rationality and irrationality, that is, the human is bounded rationally as human knowledge, imagination, and computing ability are limited in highly uncertain and extremely complex realistic decision-making environments. The application of behavioral decision theories such as prospect theory (PT) (Tversky and Kahneman 1979) and quantum probability theory (QPT) (Busemeyer et al. 2009) when conducting consensus improvement may simulate actual decision making, which can bring the decision result closer to actual decision making.

It has been verified that numerous decision deviations from traditional optimal behavior exist (such as the expected utility theory (EUT) (Neumann and Morgenstern 1944)): the uncertainty effect, reflection effect, anchoring effect, regret theory, overconfidence, and other psychological phenomena. Most of these are based on reference points. Abundant evidence shows that preferences are reference dependent, and reference points play a crucial role in explaining people's attitudes towards risk (Baillon et al. 2020). Therefore, we focus on reference-dependent decision behaviors. PT, which is the most influential descriptive theory of decisions under risk, and was proposed by Tversky and Kahneman (1979), evaluates outcomes as gains and losses from a reference point. PT can fully demonstrate the complexity and uncertainty of human decision behavior, as well as the central role of reference dependence; thus, it is introduced in our consensus modeling. The universality of PT has been verified by extensive studies in GDM with various preference forms and it has been applied in many fields through combinations with different theoretical methods in the modeling process (Ding et al. 2019b; Zhou et al. 2019; Zhu et al. 2017). Guided by PT, we prefer the adoption of the prospect value to measure consensus, which depicts individual risk preference psychology effectively and reliably. The reason is that although several identification rules assist in selecting the less contributing individuals, such as distance functions (Gong et al. 2015; Li et al. 2019), clustering (Kamis et al. 2017; $\mathrm{Wu}$ and $\mathrm{Xu}$ 2018), conflict detection (Liu et al. 2019; Ding et al. 2019a), and 
consensus evolution networks (Wu et al. 2019), these are all essentially based on similarities. In fact, although DMs provide the same evaluation of an alternative and the consensus level based on the preference similarity is sufficiently high, it is possible that individual psychological utilities will vary owing to the different reference points. However, this possibility is commonly ignored by existing works, which fit the personalized preferences and actual decision-making behaviors of DMs. Therefore, it would be significant to investigate the consensus measurement based on PT.

In general, individuals have their own preferences for selecting a reference point, and it is necessary for the reference point to be selected carefully (Dolan and Robinson 2001). The selection of the reference point is dependent on several complex factors in GDM, such as the DM preferences, decision goals, and current decision environment. In addition to the widely used crisp numbers, such as denoting the assumptive value, average value, and expectation as reference points (Ding et al. 2019b; Xu et al. 2018; Zhou et al. 2019), Wang et al. (2015) proposed a PT-based interval dynamic reference point. Moreover, Zhu et al. (2017) provided three reference points for multi-stage risk decision issues, including the expected values, positive ideal points, and development status of alternatives. Song and Zhu (2019) also proposed three reference points considering the status quo, minimum requirement, and goal. It should be noted that Baillon et al. (2020) summarized six cases of reference settings, including status quo, MaxMin, MinMax, X at Max $P$, expected value, and prospect itself (details can be found in Sect. 4.1) and explored the most common rules. In the research on decision making based on PT, the reference point is considered to change dynamically with different decision stages and scenarios in many fields. In terms of the dynamic reference point in the CRP, current methods mainly focus on the updated reference point in the new round of decision making following the change in the evaluation information (Wang et al. 2015; Gao et al. 2017); however, the reference point is supposed to be dynamic during the modification process. A challenging problem that arises in this domain is the construction of a modification model that designs the dynamic reference point along the evaluation changes to obtain the optimized adjusted evaluation.

Many alternative methods are available in the CRP for solving the determination of reference information (Kamis et al. 2017; Zhang et al. 2020). To consider the various factors comprehensively, Fan et al. (2002) insisted that it is necessary to construct decision-making models based on subjective and objective information. Subsequently, Zhang et al. (2016) extended the relevant research by simulating decision analyses based on subjective psychological characteristics and objective reference information. Song and Zhu (2019) introduced a series of decision-making methods with a cloud model based on objective and subjective information. Inspired by this method, we generate the reference information from the objective and subjective preferences, and thus, the aggregation model should be proposed. The common strategy that is used to study the integration of information from different classifications in GDM is based on classical probability theory (i.e. the law of total probability). In this process, the potential hypothesis is that the DM considers the classification paths sequentially and the decision is presented as a determinate basic state at any moment; thus, the final decision state 
is dependent on the probabilities of the basic states. However, numerous studies and experiments have proven the inapplicability of the law of total probability in classification decision making, whereas QPT can simulate real decision making effectively (Busemeyer et al. 2009; Wang et al. 2014; Wang and Busemeyer 2016). This is because the classification paths are considered simultaneously in QPT, which corresponds to real decision making. QPT can demonstrate this mode through obtaining the final decision state by the superposition of the probability amplitudes of the coexisting basic states, and the resulting interference term of the categorization on the decision making explains the violation of the law of total probability. Specifically, the interference occurs when the consciousness flows across different classification paths, which is belief superposition. QPT can elucidate many other paradoxes apart from the violation of the law of total probability (Yukalov and Sornette 2012; Aerts and Sozzo 2012), and quantum decision models have been developed to describe human decision making in different applications (Busemeyer et al. 2015; Busemeyer and Wang 2018; He et al. 2018) over the past decades (Aerts and Aerts 1995; Khrennikov 1999). It has been conclusively demonstrated that QPT has verified its applicability and superiority through many studies. Given that insufficient work has been devoted to information integration from different classifications based on QPT in the CRP, and that the integration of subjective and objective information has been verified as reasonable for actual decision making, we focus on the quantum integration of these two classifications of information. Moreover, essential concern exists regarding the determination of the interference term. The interference term plays a pivotal role in simulating decision behavior, and no unified method has been developed to determine its value. The obstacle that is overcome in this study is the exploration of the optimization value of the interference term to acquire the most acceptable personalized reference information for an individual.

Based on the motivations outlined above, this study aims to investigate a consensus mechanism with the consensus measurement based on the prospect value, the reference information integration guided by QPT, and the modification model considering the dynamic reference point. As linguistic distribution assessment has received substantial interest in describing preferences by revealing the complexity in real decision making (Zhang et al. 2018; Wu et al. 2018, 2020), this study focuses on exploring the CRP in linguistic distribution GDM. The main contributions of this proposal include three aspects:

- The concept of "attitude consistency" in the linguistic distribution context is proposed to improve the quality of the linguistic distribution assessment with higher accuracy, and it is integrated into the CRP modeling.

- The reference assessment is acquired through integrating different information with QPT, and the multi-objective programming model is constructed to obtain the value of the interference term based on the maximum individual utility.

- A dynamic reference point-oriented consensus model is constructed, the consensus measurement of which is computed with the prospect value of the alternative so as to obtain the optimized modified information in the CRP based on the best consensus level. 
The applicability and effectiveness of the proposed CRP are demonstrated by an illustrative example and a comparison discussion. It is established that the constructed consensus model can analyze the linguistic distribution information more accurately, as well as guarantee maximal individual utility. Our approach for improving the consensus involves the use of different behavior decision theories that can be applied to reflect the dynamic characteristics of GDM, and the irrational behavior and belief superposition of DMs in classification decisions.

The remainder of this paper is organized as follows: Section 2 briefly outlines several concepts relating to linguistic distribution assessment and behavioral decision theories. Section 3 introduces the acquisition of reference information, including the aggregation of subjective preferences, and the quantum integration of subjective and objective preferences. Section 4 describes the proposed dynamic reference point-based consensus model. Section 5 presents an application example to demonstrate the performance of the proposed models, along with the results analysis and a comparison with other methods according to four aspects. Finally, conclusions and future directions are provided in Sect. 6.

\section{Preliminaries}

Suppose that $M=1,2, \ldots, m$ and $N=1,2, \ldots, n$. In this study, there are $m$ DMs, denoted as $d m^{k}(k \in M)$, ranking $n$ alternatives, denoted as $A_{i}(i \in N)$ through negotiation and communication based on the respective preference information. In this section, we briefly discuss the concepts of linguistic distribution assessments and behavioral decision theories.

\subsection{Linguistic Distribution Assessment}

The relevant concepts and condition construction that are adopted in the consensus modeling of linguistic distribution assessments are described as follows:

\subsubsection{Relevant Concepts}

Zadeh (1975) innovatively proposed that the linguistic variable, which is in the form of words or sentences with a natural or artificial language, can make information evaluation more realistic and flexible. On this basis, Herrera and Herrera-Viedma (2000) introduced an ordered discrete linguistic term set (LTS) using the following mathematical expression: Let $S=\left\{S_{\alpha} \mid \alpha=0,1, \ldots, g-1, g\right\}$ be a finite and totally ordered discrete LTS with odd granularity $g+1$, where $S_{\alpha}$ represents the possible value of a linguistic variable. The set of linguistic variables with granularity 7 is as follows: $S=\left\{S_{0}\right.$ : very poor, $S_{1}$ : poor, $S_{2}$ : sightly poor, $S_{3}:$ fair, $S_{4}$ : sightly good, $S_{5}$ : good, and $S_{6}$ : very good\}. $S$ must satisfy the following characteristics with $S_{\alpha}, S_{\beta} \in S$ :

(1) The set is ordered as $S_{\alpha} \leq S_{\beta}$ if and only if $\alpha \leq \beta$.

(2) There is a negation operator: $\operatorname{Neg}\left(S_{\alpha}\right)=S_{g-\alpha}$. 
A novel linguistic information expression known as linguistic distribution assessment was presented by Zhang et al. (2014), which contains the linguistic term $S_{\alpha}$ in $S$, and each term has a corresponding distribution $\rho_{\alpha}$. The relevant definitions and operational laws are as follows:

Definition 1 (Zhang et al. 2014). Let $S$ be as before: $M=\left\{\left(S_{\alpha}, \rho_{\alpha}\right) \mid \alpha=0,1, \ldots, g\right\}$, where $S_{\alpha} \in S, \rho_{\alpha} \in[0,1], \sum_{\alpha=0}^{g} \rho_{\alpha}=1$, and $\rho_{\alpha}$ is the symbolic proportion of $S_{\alpha}$. Thus, $M$ is known as the distribution assessment of $S$. The distance measure between two arbitrary linguistic distribution assessments $M^{1}=\left\{\left(S_{\alpha}, \rho_{\alpha}^{1}\right) \mid \alpha=0,1, \ldots, g\right\}$ and $M^{2}=\left\{\left(S_{\alpha}, \rho_{\alpha}^{2}\right) \mid \alpha=0,1, \ldots, g\right\}$ is:

$$
d\left(M^{1}, M^{2}\right)=\frac{1}{2} \sum_{\alpha=0}^{g}\left|\rho_{\alpha}^{1}-\rho_{\alpha}^{2}\right|
$$

The expectation that is associated with the linguistic distribution assessment $M$ can be obtained as follows:

$$
E V(M)=\sum_{\alpha=0}^{g} \alpha \cdot \rho_{\alpha}
$$

\subsubsection{Relevant Model Construction}

Reliable and logical preference information is necessary in the GDM process. The rules should be set according to the characteristics of different evaluations to normalize the expression. For linguistic distribution assessment, the following features were explored in Zhang et al. (2018) and Wu et al. (2018, 2020):

- Succession: the linguistic terms $S_{\alpha}$ with corresponding $\rho_{\alpha} \neq 0$ should be successive. For example, $M=\left\{\left(S_{1}, 0.3\right),\left(S_{2}, 0\right),\left(S_{3}, 0.5\right),\left(S_{4}, 0.2\right)\right\}$ are not successive.

- Accuracy: the evaluation information should be as accurate as possible with few linguistic terms. For example, $M=\left\{\left(S_{\alpha}, 1\right)\right\}$ is the most precise expression because it involves only one linguistic term, whereas $M=\left\{\left(S_{0}, 0.1\right),\left(S_{1}, 0.2\right),\left(S_{3}, 0.3\right),\left(S_{4}, 0.2\right),\left(S_{5}, 0.1\right),\left(S_{6}, 0.1\right)\right\}$ is the roughest form because it contains all linguistic terms with $g=6$.

Therefore, accurate constraints can be constructed to guarantee precise, concentrated, and logical preferences:

$$
\begin{cases}\sum_{\alpha=0}^{g-1}\left|z_{\alpha+1}-z_{\alpha}\right| \leq 2 & (1-1) \\ z_{0}+z_{g} \leq 1 & (1-2) \\ \sum_{\alpha=0}^{g} z_{\alpha} \leq N & (1-3) \\ z_{\alpha}= \begin{cases}0, \rho_{\alpha}=0 \\ 1, \rho_{\alpha} \neq 0\end{cases} & (1-4) \\ \rho_{\alpha} \in[0,1] & (1-5)\end{cases}
$$


where constraints $(1-1)$ and $(1-2)$ guarantee succession by introducing the binary variable $z_{\alpha}$, which can measure whether the DM provides an evaluation on $\rho_{\alpha}$; constraint $(1-3)$ indicates the accuracy, $\sum_{\alpha=0}^{g} z_{\alpha}$ represents the number of involved terms, and a larger value of $\sum_{\alpha=0}^{g} z_{\alpha}$ has a lower accuracy. Different values for $N$ can be set according to practical decision problems and the individual preference of the DM; constraint $(1-5)$ states the range of $\rho_{\alpha}$ (details can be found in Zhang et al. (2018) and Wu et al. $(2018,2020))$.

\subsection{Behavioral Decision Theories}

In this study, we apply behavioral decision theories, including QPT and PT, to the consensus model, and the main concepts are presented below.

\subsubsection{QPT}

Busemeyer and Wang verified the rationality and superiority of quantum theory frameworks in classification decision making compared to the Markov process through experiments (Busemeyer et al. 2009; Wang and Busemeyer 2016). They held that the decision state is reflected by the wave function (probability amplitude), and the final decision state is the superposition of several basic decision states. The probability of the decision at state $r$ can be obtained if the wave function $\varphi_{r}$ is known, which is expressed as the square of the probability amplitude $\left|\varphi_{r}\right|^{2}$. On this basis, He et al. (2018) introduced QPT into multiple-attribute GDM and analyzed the opinion aggregation through different paths simultaneously. It is commonly stated that the probability of selecting alternative $A_{i}$ in the Markov process is expressed as:

$$
\bar{P}\left(A_{i}\right)=\sigma \sum_{j} P\left(C_{j}\right) P\left(A_{i} \mid C_{j}\right)
$$

where $C_{j}$ denotes the classification path $j, P\left(C_{j}\right)$ is the probability of selecting path $j$, $P\left(A_{i} \mid C_{j}\right)$ is the probability of selecting alternative $A_{i}$ with path $j$, and $\sigma$ is a normalization factor that can result in $\sum_{i} P\left(A_{i}\right)=1$. He et al. (2018) summarized the quantum probability framework to obtain the value of $P\left(A_{i}\right)$, as follows:

$$
P\left(A_{i}\right)=\sigma\left|\sum_{j} \varphi_{C_{j}} \varphi_{A_{i} \mid C_{j}}\right|^{2}
$$

where $\varphi_{C_{j}}$ is the probability amplitude of selecting path $j, \varphi\left(A_{i} \mid C_{j}\right)$ is the probability amplitude of selecting alternative $A_{i}$ with path $j$, and $\sigma$ is a normalization factor, as before. This formula indicates that the classical probability is the squared magnitude of the relevant amplitude probability. In particular, when only two paths $C_{j}$ and $C_{j^{\prime}}$ are considered:

$$
P\left(A_{i}\right)=\sigma\left|\varphi_{C_{j}} \varphi_{A_{i} \mid C_{j}}+\varphi_{C_{j^{\prime}}} \varphi_{A_{i} \mid C_{j^{\prime}}}\right|^{2}
$$


As in the quantum framework, the squared magnitude of the amplitude probability is equal to the value of multiplying the amplitude with its complex conjugate (denoted by *). Subsequently,

$$
P\left(A_{i}\right)=\sigma\left(\left|\varphi_{C_{j}} \varphi_{A_{i} \mid C_{j}}+\varphi_{C_{j^{\prime}}} \varphi_{A_{i} \mid C_{j^{\prime}}}\right|\left|\varphi_{C_{j}} \varphi_{A_{i} \mid C_{j}}+\varphi_{C_{j^{\prime}}} \varphi_{A_{i} \mid C_{j^{\prime}}}\right|^{*}\right)
$$

with

$$
\varphi_{C_{j}}=\sqrt{P\left(C_{j}\right)} e^{i \theta_{C_{j}}}, \varphi_{A_{i} \mid C_{j}}=\sqrt{P\left(A_{i} \mid C_{j}\right)} e^{i \theta_{A_{i} \mid C_{j}}}
$$

where $e^{i \theta}$ is the phase of the corresponding amplitude, and the meanings of the other symbols are the same as before. Thus,

$$
\begin{aligned}
P\left(A_{i}\right)= & \sigma\left[\left(\sqrt{P\left(C_{j}\right) P\left(A_{i} \mid C_{j}\right)} e^{i \theta_{C_{j}}}+\sqrt{P\left(C_{j^{\prime}}\right) P\left(A_{i} \mid C_{j^{\prime}}\right)} e^{i \theta_{C_{j^{\prime}}}}\right)\right. \\
& \left(\sqrt{P\left(C_{j}\right) P\left(A_{i} \mid C_{j}\right)} e^{-i \theta_{C_{j}}}+\sqrt{P\left(C_{j^{\prime}}\right) P\left(A_{i} \mid C_{j^{\prime}}\right)} e^{\left.\left.-i \theta_{j^{\prime}}\right)\right]}\right. \\
= & \sigma\left[P\left(C_{j}\right) P\left(A_{i} \mid C_{j}\right)+P\left(C_{j^{\prime}}\right) P\left(A_{i} \mid C_{j^{\prime}}\right)+\sqrt{P\left(C_{j}\right) P\left(A_{i} \mid C_{j}\right)} \sqrt{P\left(C_{j^{\prime}}\right) P\left(A_{i} \mid C_{j^{\prime}}\right)} e^{i\left(\theta_{C_{j}}-\theta_{C_{j^{\prime}}}\right)}\right. \\
& \left.+\sqrt{P\left(C_{j}\right) P\left(A_{i} \mid C_{j}\right)} \sqrt{P\left(C_{j^{\prime}}\right) P\left(A_{i} \mid C_{j^{\prime}}\right)} e^{i\left(\theta_{C_{j^{\prime}}}-\theta_{C_{j}}\right)}\right] \\
= & \sigma\left[P\left(C_{j}\right) P\left(A_{i} \mid C_{j}\right)+P\left(C_{j^{\prime}}\right) P\left(A_{i} \mid C_{j^{\prime}}\right)+2 \sqrt{P\left(C_{j}\right) P\left(A_{i} \mid C_{j}\right) P\left(C_{j^{\prime}}\right) P\left(A_{i} \mid C_{j^{\prime}}\right)} \cos \theta\right]
\end{aligned}
$$

It is clear that $2 \sqrt{P\left(C_{j}\right) P\left(A_{i} \mid C_{j}\right) P\left(C_{j^{\prime}}\right) P\left(A_{i} \mid C_{j^{\prime}}\right)} \cos \theta$ is the additional part compared with the Markov process. In QPT, $2 \sqrt{P\left(C_{j}\right) P\left(A_{i} \mid C_{j}\right) P\left(C_{j^{\prime}}\right) P\left(A_{i} \mid C_{j^{\prime}}\right)} \cos \theta$ is known as the interference term. Furthermore, $\theta=\theta_{C_{j}}-\theta_{C_{j^{\prime}}}$ represents the angle difference between the interference phase angles concerning the paths of $C_{j}$ and $C_{j^{\prime}}$. If $0 \leq \theta<\frac{\pi}{2}$, it is implied that a positive interference exists to a certain extent. If $\theta=\frac{\pi}{2}$, there is no interference term and the quantum probability framework will be equivalent to the law of total probability. If $\frac{\pi}{2}<\theta \leq \pi$, it is implied that a negative interference exists to a certain extent.

\subsubsection{PT}

The main concept of PT can be summarized as follows: (1) the outcomes are obtained by gains or losses compared to the reference point instead of the expectation calculated using the EUT. (2) DMs take risk-averse attitudes towards gains and take risk-seeking attitudes towards losses, and they exhibit more sensibility on losses than on gains. The detailed formula for the value function is as follows:

$$
v= \begin{cases}(x-\bar{x})^{\mu}, & x \geq \bar{x} \\ -\lambda(\bar{x}-x)^{\nu}, & x<\bar{x}\end{cases}
$$

where $\bar{x}$ refers to the reference point and $x$ is the actual outcome of the alternative. $\mu$ and $\nu$ are the exponent parameters relating to the gains and losses, respectively. $\lambda$ is the risk-aversion parameter, which determines a steeper characteristic of losses than 
of gains. The values of $\mu, v$, and $\lambda$ were determined through experiments and are provided in Tversky and Kahneman (1992): $\mu=0.89, \nu=0.92$, and $\lambda=2.25$.

The prospect value can guide the alternative selection; that is, the decision option with the maximum value gains the highest priority. The prospect value can be obtained through the prospect function and corresponding probability:

$$
P V=v \cdot p
$$

Table 1 Notations in proposed consensus model

\begin{tabular}{|c|c|}
\hline Notations & Meanings \\
\hline$d m^{k}$ & Decision maker $k$ \\
\hline$A_{i}$ & Alternative $i$ \\
\hline$M^{l(i)}$ & Linguistic distribution assessment of $d m^{l}$ for $A_{i}$ \\
\hline$M\urcorner k(i)$ & The rough aggregated preference (except $d m^{k}$ ) for $A_{i}$ \\
\hline$S_{\alpha}$ & Linguistic term \\
\hline$\rho_{\alpha}^{k(i)}$ & $d m^{k}$, s distribution on $S_{\alpha}$ for $A_{i}$ \\
\hline$\rho_{\alpha}^{\urcorner k(i)}$ & The rough aggregated preference (except $d m^{k}$ )'s distribution on $S_{\alpha}$ for $A_{i}$ \\
\hline$\overline{E V}^{7(i)}$ & The expected value of accurate aggregated preference (except $d m^{k}$ ) \\
\hline$E V^{-k(i)}$ & The expected value of $M^{\urcorner k(i)}$ \\
\hline$\widetilde{E V}^{k(i)}$ & The expected value of reference information of $d m^{k}$ for $A_{i}$ \\
\hline $\bar{\rho}_{\alpha}^{\urcorner k(i)}$ & The accurate aggregated preference (except $d m^{k}$ )'s distribution on $S_{\alpha}$ for $A_{i}$ \\
\hline$z_{\alpha}^{\urcorner(i)}$ & The binary variable to measure whether $\bar{\rho}_{\alpha}{ }^{k(i)}$ is equal to 0 \\
\hline$\rho_{\alpha}^{(i)}$ & Objective distribution on $S_{\alpha}$ for $A_{i}$ \\
\hline$\tilde{\rho}_{\alpha}^{k(i)}$ & Reference distribution on $S_{\alpha}$ of $d m^{k}$ for $A_{i}$ \\
\hline$P\left(C_{\text {sub }}\right)$ & The probability of consciousness flowing towards the subjective information \\
\hline$P\left(C_{o b j}\right)$ & The probability of consciousness flowing towards the objective information \\
\hline$\theta$ & Phase difference \\
\hline$\sigma^{k(i)}$ & The normalization factor in the quantum integration of $d m^{k}$ for $A_{i}$ \\
\hline$d^{k}$ & Total distance of prospect values on all alternatives for $d m^{k}$ \\
\hline$\phi$ & the prospect distance threshold \\
\hline$P V^{k(i)}$ & The prospect value of $d m^{k}$ for $A_{i}$ \\
\hline$Q_{\alpha}^{k(i)}$ & The intermediate binary variable to determine reference point \\
\hline$R^{k(i)}$ & The minimum subscript of linguistic distribution assessment for $d m^{k}$ for $A_{i}$ \\
\hline$R^{k}$ & The subscript of reference point for $d m^{k}$ \\
\hline$z_{\alpha}^{k(i)}$ & The binary variable to measure whether $S_{\alpha}$ is involved in the modified preference \\
\hline $\bar{\rho}_{\alpha}^{k(i)}$ & The modified distribution on $S_{\alpha}$ of $d m^{k}$ for $A_{i}$ \\
\hline$T_{\alpha}^{k(i)}$ & Value function of $d m^{k}$ for $A_{i}$ \\
\hline$S\left(A_{i}\right)$ & Score for $A_{i}$ \\
\hline
\end{tabular}


Notes For linguistic distribution assessment, the subscript of the linguistic term is used in the calculation of the prospect value in this study, and the distribution denotes the probability $p$ in PT and QPT.

The list of notations in the proposed consensus model are presented in Table 1.

\section{Acquisition of Reference Information}

As mentioned previously, when the identified $d m^{k}$ is suggested to make modifications, he/she will refer to several recommendations. In this study, we assume that the reference information source involves two paths: objective information and subjective information. The objective information is based on actual data that have occurred in the past from the perspective of practice. The subjective information is the provided preferences of the DMs, except for $d m^{k}$, s, which is from the perspective of consensus improvement. As introduced above, during the process of aggregating information from these two paths, an interference effect will emerge as DMs consider the two paths simultaneously (that is, there is no explicit categorization aimed at subjective and objective information prior to the decision). Therefore, the adoption of QPT to explore the integration process is more aligned with a realistic decision-making environment. It is evident that the objective information is a linguistic distribution assessment and the subjective information is composed of $m-1$ linguistic distribution assessments. It is suggested that the aggregation of subjective preferences is necessary in the first step.

\subsection{Aggregation of Subjective Preferences}

Assuming that the DMs except for $d m^{k}$ can be represented as $d m^{l}(l \in M, l \neq k)$, their preference for alternative $A_{i}$, which is denoted by $M^{l(i)}=\left\{\left(S_{\alpha}, \rho_{\alpha}^{l(i)}\right) \mid \alpha=0, \ldots, g\right\}$, is expressed by linguistic distribution assessments. According to the weighted method adopted in much of the current literature on aggregating information (Zhang et al. 2020; Jing et al. 2020) with equal individual weights, the normalized aggregated preference of all $\mathrm{dm}^{l}$ can be obtained for $A_{i}$ through Eq. (5).

$$
M^{k(i)}=\left\{\left(S_{\alpha}, \rho_{\alpha}^{\urcorner(i)}\right) \mid \alpha=0, \ldots, g\right\}=\left\{\left(S_{\alpha}, \frac{\sum_{l}^{m-1} \rho_{\alpha}^{l(i)}}{m-1}\right) \mid \alpha=0, \ldots, g\right\}
$$

Although $M^{\neg k(i)}$ is comprehensive in that it covers all of the linguistic terms included in the individual assessments, in this study, the aim of aggregating the subjective information is to provide guidance for the identified DM, and thus, the aggregated information should be sufficiently accurate to improve the reference value and to enhance the decision-making efficiency. Obviously, $M^{k(i)}$ cannot satisfy the restrictions mentioned in Sect. 2.1.2. Therefore, it is necessary to construct a model to ensure that the aggregated collective information is accurate. Constraints (1) should be included in the aggregation model. Furthermore, according to the above analysis, $N$ determines the number of linguistic terms in the assessment. In general, $N=1,2,3$, and the value of $N$ is 2 in Zhang et al. (2018) and Wu et al. (2018, 
2020). To retain as much of the original information of $d m^{l}$ as possible, let $N=3$ in this study. In this situation, a special preference with opposite attitudes cannot be ignored (e.g., $g=6)$ : $\left\{\left(S_{2}, \rho_{2}\right),\left(S_{3}, \rho_{3}\right),\left(S_{4}, \rho_{4}\right)\right\}$ with $\rho_{2}, \rho_{3}, \rho_{4} \neq 0$. As $S_{2}$ represents "sightly poor" and $S_{4}$ denotes "sightly good", this assessment is inexact, vague, and inconsistent. Specifically, we propose that the collective preferences should satisfy the following condition that is denoted as "attitude consistency" with $N=3$ to avoid opposite preferences in a linguistic distribution assessment:

- Attitude consistency: $z_{\frac{g}{2}-1}+z_{\frac{g}{2}}+z_{\frac{g}{2}+1} \leq 2$, if $N \geq 3$

The restrictions for ensuring the accuracy of the aggregation information can be constructed through combining constraints (1) and the "attitude consistency" condition with $N \geq 3$, as follows:

$$
\left\{\begin{array}{l}
\sum_{\alpha=0}^{g-1}\left|z_{\alpha+1}-z_{\alpha}\right| \leq 2 \\
z_{0}+z_{g} \leq 1 \\
\sum_{\alpha=0}^{g} z_{\alpha} \leq N \\
z_{\alpha}=\left\{\begin{array}{l}
0, \rho_{\alpha}=0 \\
1, \rho_{\alpha} \neq 0
\end{array}\right. \\
z_{\frac{g}{2}-1}+z_{\frac{g}{2}}+z_{\frac{g}{2}+1} \leq 2 \\
\rho_{\alpha} \in[0,1]
\end{array}\right.
$$

Considering that the utility of a DM is positively related to the reserving proportion of original information (Gong et al. 2015), the objective with the minimum deviation between the initial individual assessment and aggregated information can be constructed, which contains two aspects: one focuses on the minimum distribution deviation on the same linguistic term:

$$
\operatorname{Min} \sum_{\alpha=0}^{g}\left|\rho_{\alpha}^{c}-\rho_{\alpha}\right|
$$

and the other concerns the minimum expectation deviation:

$$
\operatorname{Min}\left|E V^{c}-E V\right|
$$

where $\rho_{\alpha}^{c}$ and $E V^{c}$ are the collective information. The reason for analyzing two objectives simultaneously lies in the fact that the same distribution deviation may take different expected values, and the same expectation deviation may originate from different linguistic distributions. The advantages of this operation can be elucidated by using certain examples. Assume that $\left\{\left(S_{1}, 0.2\right),\left(S_{2}, 0.3\right),\left(S_{3}, 0.35\right),\left(S_{4}, 0.15\right)\right\}$ with expectation $E V=2.45$ must aggregate if only the minimum distribution deviation on the same linguistic term expressed by the first objective function in model (6) is constructed. Several assessments with the same distribution deviation of 0.3 are obtained: $\left\{\left(S_{1}, 0.2\right),\left(S_{2}, 0.45\right),\left(S_{3}, 0.35\right)\right\},\left\{\left(S_{1}, 0.35\right),\left(S_{2}, 0.3\right),\left(S_{3}, 0.35\right)\right\}$, and $\left\{\left(S_{1}, 0.2\right),\left(S_{2}, 0.3\right),\left(S_{3}, 0.5\right)\right\}$; however, the expectations are 2.15, 2, and 2.3 , respectively, and it is obvious that 2.3 is the closest to 2.45 . Therefore, $\left\{\left(S_{1}, 0.2\right),\left(S_{2}, 0.3\right),\left(S_{3}, 0.5\right)\right\}$ is the most optimized solution. If only the 
minimum expectation expressed by the second objective function in model (6) is considered, several assessments with the same expectation of 2.45 are obtained: $\left\{\left(S_{1}, 0.125\right),\left(S_{2}, 0.3\right),\left(S_{3}, 0.575\right)\right\},\left\{\left(S_{2}, 0.55\right),\left(S_{3}, 0.45\right)\right\}$; however, the total distribution deviations are 0.45 and 0.7 . Thus, $\left\{\left(S_{1}, 0.125\right),\left(S_{2}, 0.3\right),\left(S_{3}, 0.575\right)\right\}$ is the most optimized solution. Taken together, these results suggest that the construction of a multi-objective programming model (denoted as model (6)) is necessary for aggregating information. The priority for the objective functions depends on the behavioral preference of the individual.

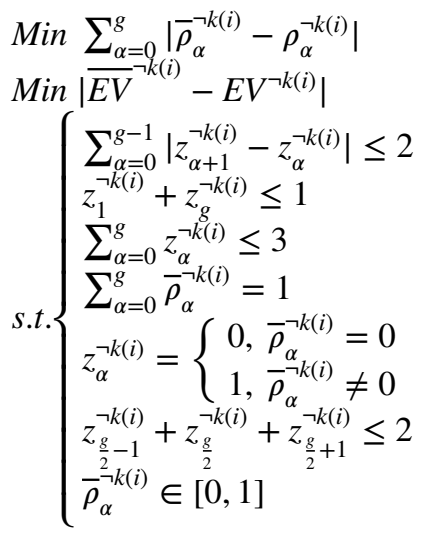

where $\bar{\rho}_{\alpha}^{\urcorner(i)}$ represents the accurate aggregated distribution of all DMs, except for $d m^{k}$ on $S_{\alpha}$ for $A_{i}, \rho_{\alpha}^{\urcorner(i)}$ denotes the rough aggregated distribution of all DMs, except for $d m^{k}$ on $S_{\alpha}$ for $A_{i}$ through Eq. (5), $\overline{E V}^{k(i)}$ is the accurate aggregated expectation of all DMs, except for $d m^{k}$ for $A_{i}$, and $E V^{\urcorner k(i)}$ denotes the rough aggregated expectation of all DMs except for $d m^{k}$ for $A_{i} . z_{\alpha}^{\urcorner(i)}$ is a binary variable to measure whether $S_{\alpha}$ is involved in the accurate aggregated preference. Through model (6), the accurate subjective reference preference of $d m^{k}$ for $A_{i}$ can be obtained as $\left\{\left(S_{\alpha}, \bar{\rho}_{\alpha}{ }^{k(i)}\right)\right\}$.
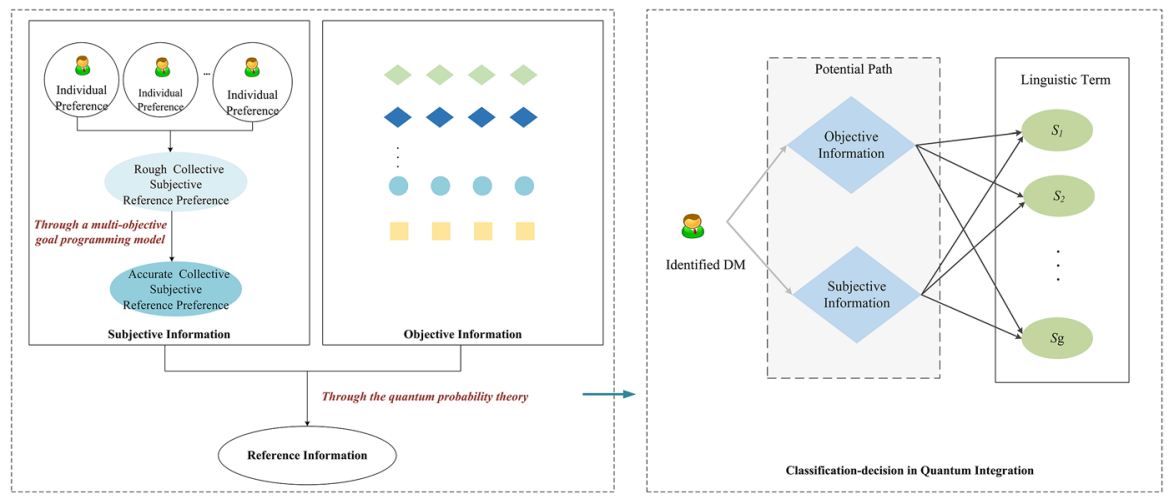

Fig. 1 Formation of reference information 


\subsection{Reference Information Acquired Through Quantum Integration of Subjective and Objective Preferences}

As mentioned previously, the identified $d m^{k}$ combines the aggregated collective subjective preference that is denoted as $\left\{\left(S_{\alpha}, \rho_{\alpha}^{\urcorner k(i)}\right)\right\}$ in Sect. 3.1, and the objective preference for $A_{i}$, denoted by $\left\{\left(S_{\alpha}, \rho_{\alpha}^{(i)}\right)\right\}$, to obtain the reference information. Fig. 1 depicts the components of the reference information and the framework of the decision classification in the proposed quantum integration: the identified individual considers the information from the objective data and subjective evaluations simultaneously, and the consciousness flowing towards the objective and subjective information is in a superposition, which reflects the uncertainty, conflict, and ambiguity of cognitive decision behavior. Finally, the distribution of each linguistic term can be acquired. In this section, we construct a multi-objective programming model to demonstrate how acceptable reference information is formed based on QPT.

Considering that less modification, more satisfied DMs (Xu et al. 2021; Jing et al. 2020; Liu et al. 2020), and reference information guide the adjustment, the identified DM may prefer that the reference information is as close to the original information as possible in the quantum integration process. Similar to the objective functions set in model (6), both the distributions on the linguistic terms, and the preferences of the expectations of the original and reference information of the individual should be considered, while QPT is applied to the simulation of the reference distribution on each linguistic term. Moreover, as the aggregated reference information is obtained by combining the subjective and objective information, it should not exceed the subjective and objective information. Driven by the above analysis, the following model can be constructed to obtain the identified reference information of $d m^{k}$ for $A_{i}$, and a detailed explanation is provided.

$$
\begin{aligned}
& \operatorname{Min} \sum_{\alpha=0}^{g}\left|\widetilde{\rho}_{\alpha}^{k(i)}-\rho_{\alpha}^{k(i)}\right| \\
& \operatorname{Min}\left|\widetilde{E V}^{k(i)}-E V^{k(i)}\right|
\end{aligned}
$$

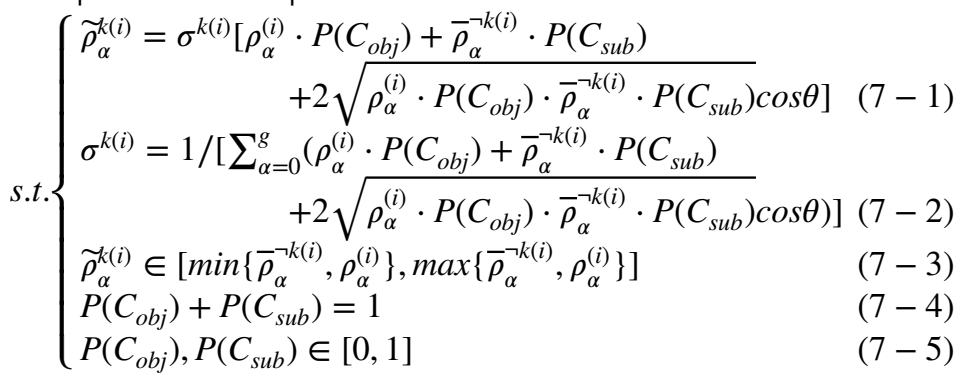

where $\widetilde{\rho}_{\alpha}^{k(i)}$ and $\rho_{\alpha}^{k(i)}$ represent the reference distributions of $d m^{k}$ and the original evaluation of $d m^{k}$ on $S_{\alpha}$ for $A_{i}$, respectively. Furthermore, $\widetilde{E V}^{k(i)}$ and $E V^{k(i)}$ denote the expectations of the reference and original information, respectively. The minimized objective functions mean that the distance between the reference information and original information of the identified DM should be as small as possible, whether the distance is measured from the perspective of the distribution on the linguistic term (expressed by the first objective function) or the expectation value of the preference 
(expressed by the second objective function). The probability of consciousness flowing towards the objective information (that is, the probability of selecting the objective information), which is denoted by $P\left(C_{o b j}\right)$, as well as the probability of consciousness flowing towards the subjective information (that is, the probability of selecting the subjective information), which is denoted as $P\left(C_{s u b}\right)$, both freely take a value in the interval $[0,1]$ and $P\left(C_{o b j}\right)+P\left(C_{\text {sub }}\right)=1$ (as revealed by $(7-4)$ and $(7-5))$. Specifically, if $P\left(C_{o b j}\right)=1$, this means that the identified DM considers the objective information only, and he/she will refer to the subjective information only when $P\left(C_{\text {sub }}\right)=1$. In these situations, only one path exists, and correspondingly, the interference effect disappears. Based on Eq. (2) regarding the quantum integration of information from two paths, constraint $(7-1)$ holds, which determines the distribution of the reference information on $S_{\alpha}$, and $\sigma^{k(i)}$ is the normalization factor, the calculation formula for which is provided as constraint $(7-2)$. As the reference information is formed through objective and accurate subjective information, the reference information should not be less than their minimum or exceed their maximum; hence, the third constraint holds. model (7) can be solved by Gurobi 9.1.0, the optimized reference preference expressed by $\left\{\left(S_{\alpha}, \widetilde{\rho}_{\alpha}^{k(i)}\right)\right\}$, and the probability of consciousness flowing towards the objective and subjective information; subsequently, the optimized difference between the interference phase angles, which is denoted as $\theta$, can be obtained. When a moderator exists in the decision making, his/her role is to prompt the consensus reaching, and he/she may suggest that the identified DM refer to a certain classification with a specific probability. In this situation, the optimized $\left\{\left(S_{\alpha}, \tilde{\rho}_{\alpha}^{k(i)}\right)\right\}$, and $\theta$ can be determined based on the maximum individual utility.

\section{Consensus Modeling with Dynamic Reference Point}

In the CRP, the identified DM will make some modifications based on his/her initial opinion once the reference information has been formed. Although it is possible that the preference information that is provided by the DMs initially exhibits an evident difference, inspired by the PT introduced in Sect. 2.2.2, DMs can seek a unified opinion to a certain extent if the prospect value that is obtained based on the reference point is similar. In detail, the consensus of $\mathrm{dm}^{k}$ and $d m^{l}$ depends on the similarity between the prospect value of $d m^{k}$, denoted as $P V^{k}$, and the prospect value of $d m^{l}$, denoted as $P V^{l}$ (where the prospect value is obtained based on the reference point and preference assessment of $d m^{k}\left(d m^{l}\right)$, rather than the similarity between the assessments of $d m^{k}$ and $d m^{l}$ in the traditional model. Moreover, the psychological expectation of the identified DM who is persuaded to make modifications may change during the communication and negotiation.

Hence, in this section, we present an approach for consensus improvement considering the dynamic reference point. It is composed of three steps: (1) reference point setting, (2) the identification rule, and (3) the consensus model. 


\subsection{Reference Point Setting}

In this study, we adopt the most preferred rule, as verified experimentally by Baillon et al. (2020); that is, the status quo and a security level (the maximum of the minimal outcomes of the prospects in a choice). Considering that the status quo is dependent on the current realistic decision environment, it is easy to portray it as a specific number, whereas the security level is reflected by the evaluation information and is dynamic in the negotiation process. Therefore, the security level is analyzed in this study. The security level can be expressed by MaxMin, which posits that in a comparison between two prospects, the DM will focus on the minimum value of the two prospects and take the maximum as the reference point. Using the linguistic distribution assessment as an example, in a comparison between $\left\{\left(S_{2}, 0.2\right),\left(S_{3}, 0.8\right)\right\}$ and $\left\{\left(S_{4}, 0.4\right),\left(S_{5}, 0.6\right)\right\}$, the minimum outcomes are $S_{2}$ and $S_{4}$, respectively. MaxMin implies that the DM will take $S_{4}$ as the reference point and will view $S_{5}$ as a gain, and $S_{2}$ and $S_{3}$ as losses (Baillon et al. 2020).

Notes According to Baillon et al. (2020), six reference point rules have been specified (the remaining four rules apart from status quo and MaxMin are listed as follows). This study only explores one of the most common reference points; the consensus modeling based on the other setting rules is similar to that of the constructed method.

- MinMax means that the DM takes the minimum of the maximum outcomes as his/ her reference point.

- $X$ at Max $P$ means the DM takes the outcome with the highest probability as his/her reference point.

- Expected Value means that the reference point is prospect specific.

- Prospect Itself specifies a stochastic reference point.

Considering $\left\{\left(S_{2}, 0.2\right),\left(S_{3}, 0.8\right)\right\}$ and $\left\{\left(S_{4}, 0.9\right),\left(S_{5}, 0.1\right)\right\}$ as an example, $S_{3}$ is selected as the reference point for MinMax, and $S_{4}$ is selected as the reference point for Xat Max P. Expected Value and Prospect Itself depend on the willingness of the DM in actual decision making.

\subsection{Identification Rule}

This rule helps to identify the DMs who contribute less to the consensus; that is, those whose total prospect value on all alternatives is far from that of the others. A prospect distance threshold $\phi$ can be used to identify the preferences that should be modified. The total distance of the prospect values on all alternatives for $d m^{k}$, which is denoted as $d^{k}$, can be calculated by

$$
d^{k}=\sum_{i}^{n} \sum_{l, l \neq k}^{m}\left|P V^{k(i)}-P V^{l(i)}\right|
$$


where $P V^{k(i)}$ is calculated using Eq. (4) and it represents the prospect value of $d m^{k}$ for $A_{i}$. DMs with an insufficient distance are listed in descending order (the DMs with $d^{k}>\phi$ ); this is the order that is followed by the model for DMs to make modifications. Without loss of generality, in this model, the assessment of only one DM requires adjustment at each iteration. As each modification improves the collective consensus (the consensus measurement should be conducted again following each modification iteration), it is possible that the DMs in the "waiting list" for modification may no longer be required to make modifications. This operation follows the principle that fewer modified DMs is preferable (the numerical example can explain this principle clearly in Sect. 5: $d m^{1}$ and $d m^{2}$ are asked for adjustment initially, but the consensus is reached after the modification of only $d m^{1}$, and there is no longer a need for $d m^{2}$ to make modifications).

\subsection{Consensus Model}

As stated previously, the consensus in this study focuses on seeking an approximately unanimous prospect value. Therefore, the distance of the prospect value between $\mathrm{dm}^{k}$ and other DMs should be as small as possible. The following minimum objective function holds:

$$
\operatorname{Min} d^{k}=\sum_{i=1}^{n} \sum_{l, l \neq k}^{m}\left|P V^{k(i)}-P V^{l(i)}\right|
$$

In PT, it is clear that the calculation of the prospect value is dependent on the reference point. As individual preference information is dynamic during the CRP, there may be changes in the reference point. For example, if there are two linguistic distribution assessments, namely $\left\{\left(S_{2}, 0.2\right),\left(S_{3}, 0.8\right)\right\}$ and $\left\{\left(S_{4}, 0.4\right),\left(S_{5}, 0.6\right)\right\}$, MaxMin implies that DM will take $S_{4}$ as the reference point; if the second assessment is modified as $\left\{\left(S_{4}, 0.3\right),\left(S_{5}, 0.7\right)\right\}$, the reference point is still $S_{4}$, but if the second assessment is modified as $\left\{\left(S_{3}, 0.3\right),\left(S_{4}, 0.7\right)\right\}$, the reference point becomes $S_{3}$. Therefore, we integrate the dynamic reference point into the CRP in an attempt to construct a dynamic reference point-oriented consensus model. A mathematical representation of the reference point needs to be provided. As introduced above, $d m^{k}$ will first select the minimum evaluation that is involved in the linguistic distribution assessment for all alternatives, and thereafter select the maximum among these as the reference point. We first use $R^{k(i)}$ to denote the subscript of the minimum linguistic term on each $A_{i}$ of $d m^{k}$. The following Eqs. (10), and (11) are provided to achieve the value of $R^{k(i)}$ by introducing a binary variable $Q_{\alpha+1}^{k(i)}$.

$$
\begin{gathered}
Q_{\alpha+1}^{k(i)}=\left\{\begin{array}{c}
0, z_{\alpha+1}^{k(i)}-z_{\alpha}^{k(i)} \leq 0, \quad \alpha=0,1, \ldots, g-1 \\
1, z_{\alpha+1}^{k(i)}-z_{\alpha}^{k(i)}>0, \quad \alpha=0,1, \ldots, g-1
\end{array}\right. \\
R^{k(i)}=\sum_{\alpha=0}^{g-1}(\alpha+1) Q_{\alpha+1}^{k(i)}, \quad i=1,2, \ldots, n
\end{gathered}
$$


where $z_{\alpha}^{k(i)}$ has the same meaning as $z_{\alpha}$ in constraints (1) for measuring whether $S_{\alpha}$ is involved in the linguistic distribution assessment provided by $d m^{k}$ for $A_{i}$. $Q_{\alpha}^{k(i)}$ can aid in identifying the minimum $S_{\alpha}$ with a distribution $\rho_{\alpha}>0$ of $d m^{k}$ for $A_{i}$. The proof is provided as follows.

\section{Proof}

- General case:

(1) If only one linguistic term is involved, namely $\left\{\left(S_{\beta+1}, 1\right)\right\}(\beta=0,1, \ldots$, $g-1), z_{\beta+1}=1, z_{\beta}=0$, and only $Q_{\beta+1}^{k(i)}=1 \mathrm{using}$ Eq. (10). Hence, $R^{k(i)}=\beta+1$. The proof is completed.

(2) If more than one linguistic term is involved, and we assume that the minimum term is $S_{\beta}$ and the maximum term is expressed by $S_{\beta^{\prime}}$ $\left(\beta<\beta^{\prime}, \beta, \beta^{\prime}=0,1, \ldots, g\right), z_{0}, z_{1}, \ldots, z_{\beta-1}=0$ and $z_{\beta}, z_{\beta+1}, \ldots, z_{\beta^{\prime}-1}, z_{\beta^{\prime}}=1$, and only $Q_{\beta}^{k(i)}=1$ using Eq. (10). Hence, $R^{k(i)}=\beta$. The proof is completed.

- Special case:

if the minimum term is $S_{0}, z_{0}=1$. Regardless of the number of linguistic terms that are included in this evaluation, all $Q_{\alpha}^{k(i)}=0$ by Eq. (10). Hence, $R^{k(i)}=0$. The proof is completed.

Subsequently, the maximum value among $R^{k(i)}$ is the reference point and it can be determined using Eq. (12).

$$
R^{k}=\max \left\{R^{k(i)} \mid i=1,2, \ldots, n\right\}
$$

Notes The reason that we do not use $\min \left\{\alpha * z_{\alpha}^{k(i)} \mid \alpha=0, \ldots, g\right\}$ to determine the minimum subscript value of $d m^{k}$ for each alternative is that the minimum value is always equal to 0 .

Equations (9) to (12) realize the dynamic characteristics of the reference point in the modification acquisition process. Obviously, the adjusted preference is expressed by the linguistic distribution, and thus, should satisfy the "Succession," "Accuracy," and "Attitude consistency" as introduced in Sects. 2.1.2 and 3.1. Moreover, the renewed information should locate between the reference and initial information, which is similar to the adjustment rule proposed by Dong et al. (2016). The mathematical expression is as follows:

$$
\bar{\rho}_{\alpha}^{k(i)} \in\left[\min \left\{\widetilde{\rho}_{\alpha}^{k(i)}, \rho_{\alpha}^{k(i)}\right\}, \max \left\{\tilde{\rho}_{\alpha}^{k(i)}, \rho_{\alpha}^{k(i)}\right\}\right]
$$

where $\bar{\rho}_{\alpha}^{k(i)}$ is the modified distribution on $S_{\alpha}$ of $d m^{k}$ for $A_{i}$.

Given that the consensus should be improved as much as possible within the acceptable range of DMs, based on the above analysis, the optimized modification model can be constructed as follows: 


$$
\begin{aligned}
& \operatorname{Min} \sum_{i=1}^{n} \sum_{l, l \neq k}^{m}\left|P V^{k(i)}-P V^{l(i)}\right|
\end{aligned}
$$

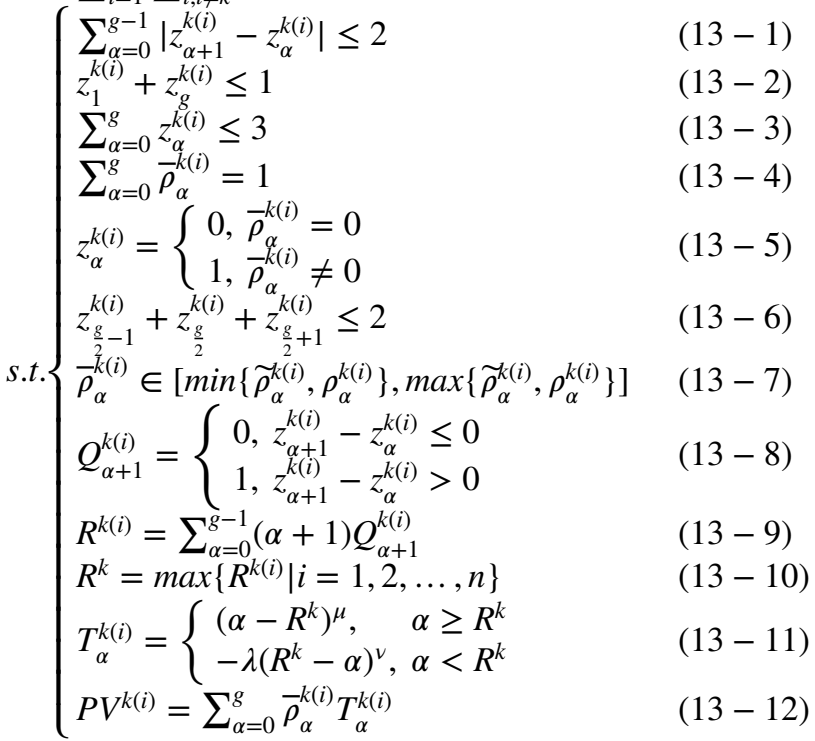

where the objective function hopes the prospect values of DMs can be as similar to each other as possible. Constraints $(13-1)-(13-6)$ are the same as those in model (6), thereby ensuring the rationality of the optimal preference. Constraint $(13-7)$ limits the modification range by the reference information obtained through model (7), which can prevent consensus retrogression as it contains the original information of the identified DM. Constraints $(13-8)-(13-10)$ determine the dynamic reference point and constraints $(13-11)-(13-12)$ are the calculation formulas for the PT. Model (13) can be solved by Gurobi 9.1.0, and the modified preference expressed by $\left\{\left(S_{\alpha}, \bar{\rho}_{\alpha}^{k(i)}\right)\right\}$ will be obtained in this manner.

Following each modification, the prospect distance must be calculated to verify whether it is no more than the predefined threshold $\phi$. If $d^{k}>\phi$ exists, the CRP needs to be conducted again; the ending condition of the CRP is each $d^{k} \leq \phi$.

\subsection{Alternative Ranking}

Once the CPR ends, an alternative ranking and selection process is conducted. The score of alternative $A_{i}$ is computed by $S\left(A_{i}\right)=\frac{1}{m-1} \sum_{k}^{m} P V^{k(i)}$, which means that for the average prospect value of $A_{i}$, a better $S\left(A_{i}\right)$ results in a better alternative $A_{i}$. The algorithm of the proposed CRP is provided in Table 2. 
Table 2 Algorithm of proposed CRP

Algorithm: The dynamic reference point-oriented CRP with the reference information integrated based on quantum probability theory.

Input: The prospect distance threshold $\phi$ to measure consensus; the original preference information provided by DMs and the objective information of alternatives

Output: The modified preference information; the alternative ranking

Step 1: $\quad$ Apply Eq.(8) to identify all $d m^{k}$ with $d^{k}>\phi$, that is who need modification;

Step 2: $\quad$ Apply Eq.(5) and model (6) to aggregate subjective information except the identified $d m^{k}$, denoted as $\left\{\left(S_{\alpha}, \bar{\rho}_{\alpha}^{\urcorner k(i)}\right)\right\}$;

Step 3: $\quad$ Apply model (7) to integrate the subjective and objective information based on quantum decision theory, denoted as $\left\{\left(S_{\alpha}, \widetilde{\rho}_{\alpha}^{k(i)}\right)\right\}$;

Step 4: $\quad$ Apply model (13) to obtain the modified preference of $d m^{k}$, denoted as $\left\{\left(S_{\alpha}, \bar{\rho}_{\alpha}^{k(i)}\right)\right\}$;

Step 5: $\quad$ Compute Eq.(8) again, if $d^{k}>\phi$ exists, then return to Step 2; otherwise, the CRP ends, the score of $A_{i}$ is computed as $S\left(A_{i}\right)$, the better $S\left(A_{i}\right)$, the better $A_{i}$;

Step 6: $\quad$ End

\section{Application Example}

In this section, we illustrate the proposed consensus model through a numerical example and analyze the effects of consciousness flowing towards different information paths on the results. Furthermore, we discuss the advantages of the consensus mechanism from several comparison viewpoints.

\subsection{Numerical Calculation}

In this subsection, we provide a numerical example about the contingency plan for the selection of designated hospitals through the above consensus process.

\subsubsection{Background}

COVID-19 continued to spread globally in 2020, and it still affects the normal travel and production activities of humans. Although the epidemic is now well under control in most areas, mutant viruses are beginning to emerge. Therefore, emergency response plans for the epidemic need to be drawn up in advance to ensure that timely and effective control measures can be taken in the event of an outbreak to ensure the safety of lives and maintain the stability of social life. The selection problem of the designated hospitals belongs to the emergency plan of the government for fighting the epidemic. A designated hospital refers to a hospital that intensively treats confirmed and suspected cases during an epidemic. Such hospitals usually have adequate professional medical care and a strong medical ability to deal with COVID19. The purpose of establishing designated hospitals is to ensure that patients can receive timely and effective treatment, while also reducing the possibility of widespread infection caused by distributed therapy. Hence, designated hospitals can play a significant role in recovery from the epidemic. Multiple factors should be considered when selecting the optimal hospital as the designated facility, such as the 
Table 3 Original preference information of DMs in candidate hospitals

\begin{tabular}{llll}
\hline & $d m^{1}$ & $d m^{2}$ & $d m^{3}$ \\
\hline$A_{1}$ & $\left\{\left(S_{1}, 0.5\right),\left(S_{2}, 0.3\right),\left(S_{3}, 0.2\right)\right\}$ & $\left\{\left(S_{1}, 0.4\right),\left(S_{2}, 0.6\right)\right\}$ & $\left\{\left(S_{3}, 0.7\right),\left(S_{4}, 0.3\right)\right\}$ \\
$A_{2}$ & $\left\{\left(S_{4}, 0.6\right),\left(S_{5}, 0.4\right)\right\}$ & $\left\{\left(S_{2}, 0.3\right),\left(S_{3}, 0.4\right),\left(S_{4}, 0.3\right)\right\}$ & $\left\{\left(S_{4}, 0.2\right),\left(S_{5}, 0.3\right),\left(S_{6}, 0.5\right)\right\}$ \\
$A_{3}$ & $\left\{\left(S_{3}, 0.4\right),\left(S_{4}, 0.5\right),\left(S_{5}, 0.1\right)\right\}$ & $\left\{\left(S_{3}, 0.7\right),\left(S_{4}, 0.3\right)\right\}$ & $\left\{\left(S_{5}, 0.6\right),\left(S_{6}, 0.4\right)\right\}$ \\
\hline
\end{tabular}

Table 4 Prospect values of DMs on candidate hospitals

\begin{tabular}{lccc}
\hline & $d m^{1}$ & $d m^{2}$ & $d m^{3}$ \\
\hline$A_{1}$ & -4.82 & -3.05 & -3.66 \\
$A_{2}$ & 0.4 & -0.38 & 0.05 \\
$A_{3}$ & -0.8 & 0.3 & 0.4 \\
\hline
\end{tabular}

medical level (including instrument sufficiency, physician specialization, and medical experience), management level (including regulation standardization and logistical support), and flexibility of medical treatment (the effect on other types of patient visits). The selection problem of designated hospitals is a GDM issue.

Consider a situation in which we assume that three experts (DMs) are denoted as $d m^{1}, d m^{2}$, and $d m^{3}$, which should provide a ranking priority for three hospitals: $A_{1}, A_{2}$, and $A_{3}$. Moreover, there is a need for a uniform ranking of alternatives following communication and consultation. It is assumed that experts will adopt linguistic distributed assessments to provide preference information for each hospital, so as to express their evaluations comprehensively and completely using more than one linguistic term, owing to the diversity of the criteria listed above, as well as the complexities and vagaries of epidemic situations. Given that experts have different evaluation behavior habits and individual expectations differ for a particular criterion, it is appropriate to focus on the consensus-building process based on psychological behavior characteristics. After identifying the expert who is furthest from the opinion of the group, this expert needs to adjust his/her preference to improve the consensus level. During this process, he/she will also refer to the subjective evaluation information of other experts and the objective information of each hospital based on the past medical treatment and processing ability in response to similar emergencies, while retaining their own opinions to a certain extent. The original preference information of experts on the three candidate hospitals is presented in Table 3.

\subsubsection{Consensus Measurement}

According to the reference point rule that is adopted in this study, it is easy to determine that the reference point for $d m^{1}$ is $S_{4}$, for $d m^{2}$ is $S_{3}$, and for $d m^{3}$ is $S_{5}$. Based on Sect. 2.2.2, the prospect values of the DMs on the candidate hospitals can be calculated as per Table 4 . 
Table 5 Distances of prospect values of DMs on candidate hospitals

\begin{tabular}{llll}
\hline & $d m^{1}$ & $d m^{2}$ & $d m^{3}$ \\
\hline$A_{1}$ & 2.93 & 2.37 & 1.77 \\
$A_{2}$ & 1.13 & 1.2 & 0.78 \\
$A_{3}$ & 2.3 & 1.2 & 1.3 \\
Total distance & 6.36 & 4.77 & 3.85 \\
\hline
\end{tabular}

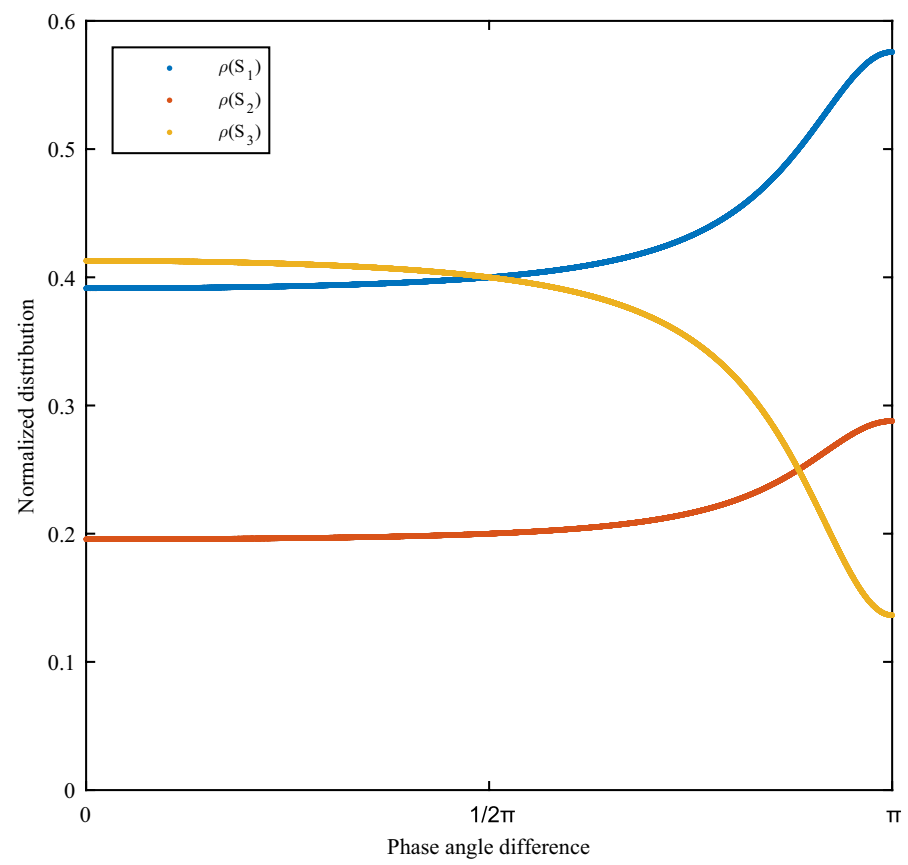

Fig. 2 Normalized distribution along phase angle difference changes on $A_{1}$

According to the identification rule, the distances of the prospect values of the DMs on the candidate hospitals are listed in Table 5 through Eq. (8). If $\phi=4.5$ in this study, $d m^{1}$ and $d m^{2}$ are suggested to make modifications successively.

\subsubsection{Reference Information Based on Quantum Integration}

As introduced above, for $d m^{1}$, the subjective reference information is obtained from $d m^{2}$ and $d m^{3}$. The rough and accurate subjective collective information can be obtained as per Table 6 through Eq. (5) and model (6), respectively. Figures 2,3 , and 4 present the normalized distributions of the linguistic terms along $\theta$ changes in the process of integrating the objective and subjective reference information based on QPT on $A_{1}, A_{2}$, and $A_{3}$ for $d m^{1}$, respectively. If the moderator suggests $P\left(C_{o b j}\right)=P\left(C_{\text {sub }}\right)=0.5$ in this example (i.e. the subjective and objective 


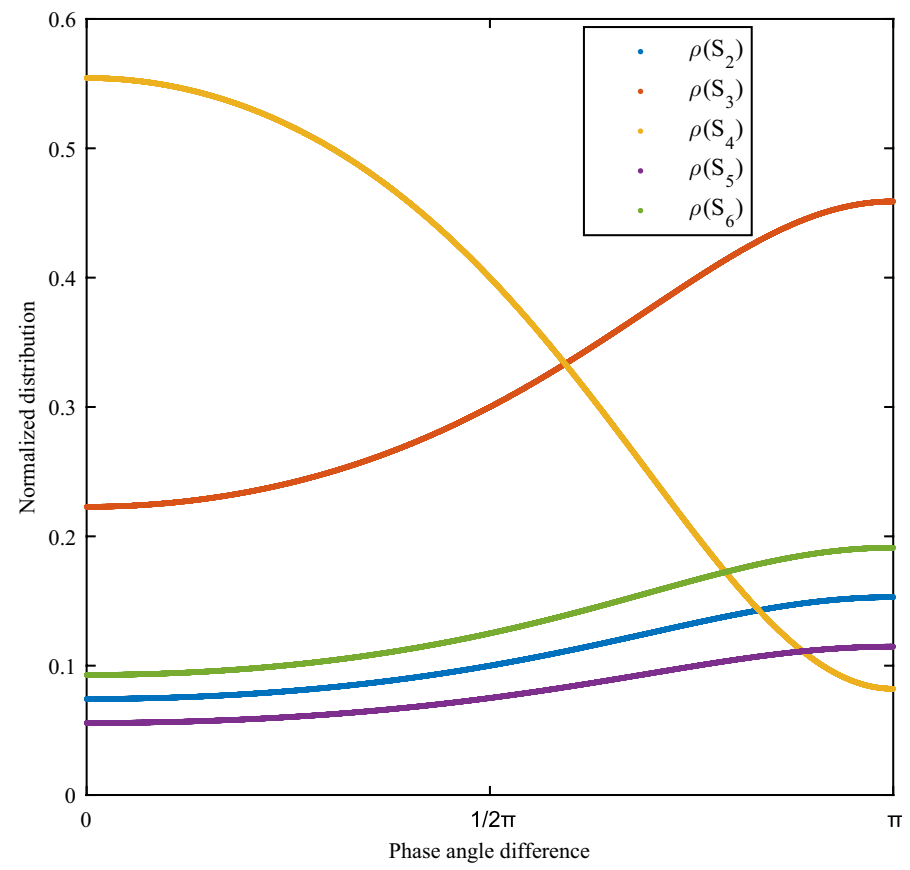

Fig. 3 Normalized distribution along phase angle difference changes on $A_{2}$

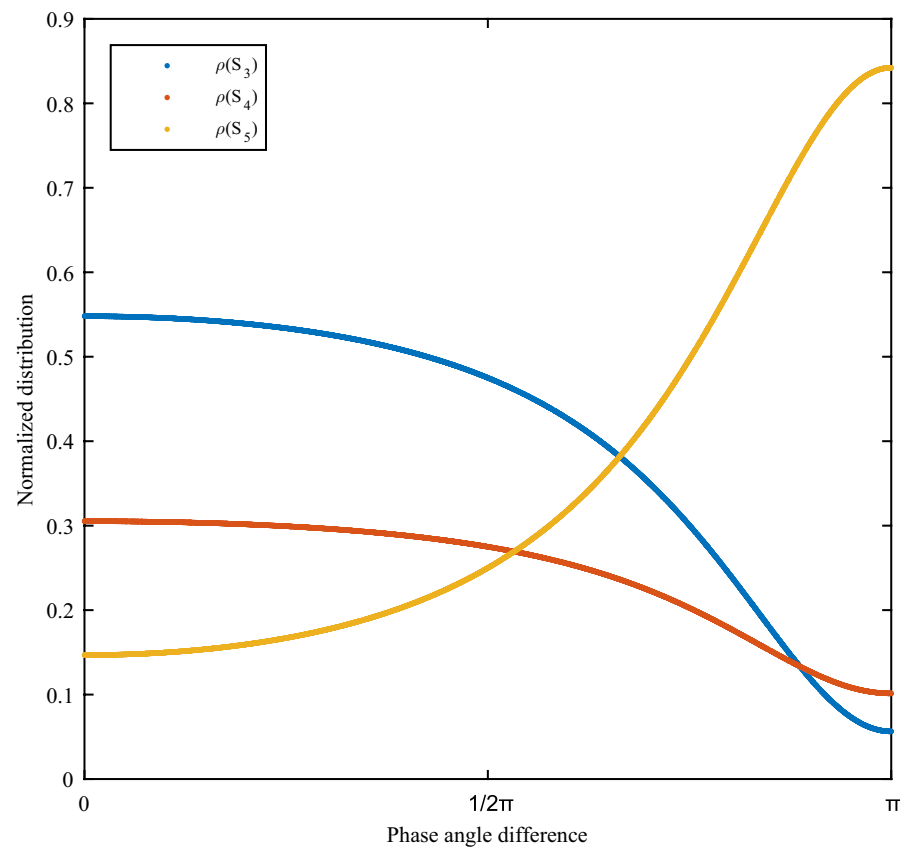

Fig. 4 Normalized distribution along phase angle difference changes on $A_{3}$ 


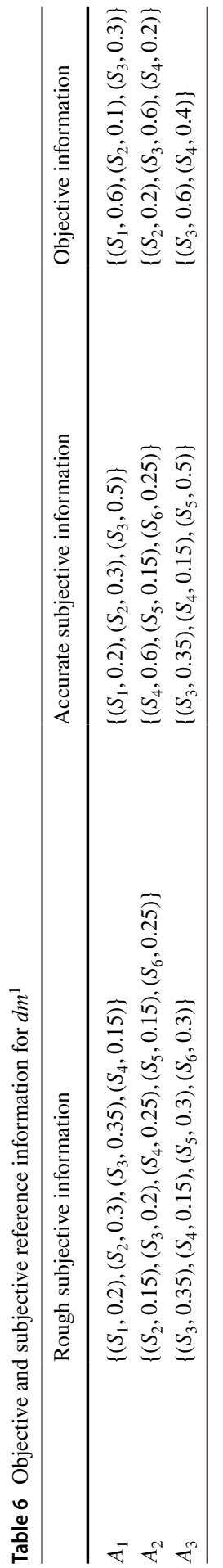


information is considered equally), through model (7), the optimized reference information for $d m^{1}$ on the alternatives can be obtained, as illustrated in Table 7. According to Table 7 , in the process of integrating the preference for $A_{1}, \theta=150^{\circ}$, a relative heavy negative interference appears. For $A_{2}$ and $A_{3}, \theta=0^{\circ}$, the interference is positive. Subsequently, the distribution range of the modification information for $d m^{1}$ based on the original information and reference information presented in Table 7 can be concluded as per Table 8 (for model (6) and model (7), the priority of the objective function is assigned for the distribution on each linguistic term in this example).

\subsubsection{Modification Information}

Using model (13), the modified preference information of $d m^{1}$ can be optimized with the adjusted reference point $S_{3}$. The relevant modifications and corresponding prospect values are displayed in Table 9. Guided by the designed consensus mechanism, the distance between the prospect values should be recalculated, as per Table 10 . Evidently, all $d^{k}$ are less than $\phi$ and the CRP ends, and there is no need for $d m^{2}$ to make a modification. This example requires only one iteration.

\subsubsection{Alternative Ranking}

The scores of the alternatives can easily be obtained: $S\left(A_{1}\right)=-3.17, S\left(A_{2}\right)=0.2$, and $S\left(A_{3}\right)=0.413$. Hence, the ranking of the alternatives is $A_{3}>A_{2}>A_{1}$. Hospital

Table 7 Reference information for $d m^{1}$

\begin{tabular}{lll}
\hline Reference information & $\begin{array}{l}\text { Phase angle } \\
\text { difference } \theta\end{array}$ \\
\hline$A_{1}\left\{\left(S_{1}, 0.47\right),\left(S_{2}, 0.23\right),\left(S_{3}, 0.3\right)\right\}$ & $150^{\circ}$ \\
$A_{2}\left\{\left(S_{2}, 0.07\right),\left(S_{3}, 0.22\right),\left(S_{4}, 0.55\right),\left(S_{5}, 0.06\right),\left(S_{6}, 0.1\right)\right\}$ & $0^{\circ}$ \\
$A_{3}$ & $\left\{\left(S_{3}, 0.55\right),\left(S_{4}, 0.3\right),\left(S_{5}, 0.15\right)\right\}$ & $0^{\circ}$ \\
\hline
\end{tabular}

Table 8 Distribution range of modification information for $d m^{1}$

\begin{tabular}{llllllll}
\hline & $\rho_{0}^{k(i)}$ & $\rho_{1}^{k(i)}$ & $\rho_{2}^{k(i)}$ & $\rho_{3}^{k(i)}$ & $\rho_{4}^{k(i)}$ & $\rho_{5}^{k(i)}$ & $\rho_{6}^{k(i)}$ \\
\hline$A_{1}$ & 0 & {$[0.47,0.5]$} & {$[0.23,0.3]$} & {$[0.2,0.3]$} & 0 & 0 & 0 \\
$A_{2}$ & 0 & 0 & {$[0,0.07]$} & {$[0,0.22]$} & {$[0.55,0.6]$} & {$[0.06,0.4]$} & {$[0,0.01]$} \\
$A_{3}$ & 0 & 0 & 0 & {$[0.4,0.55]$} & {$[0.3,0.5]$} & {$[0.1,0.15]$} & 0 \\
\hline
\end{tabular}

Table 9 Modified preference information for $d m^{1}$

\begin{tabular}{llc}
\hline & Modified information & Prospect value \\
\hline$A_{1}$ & $\left\{\left(S_{1}, 0.5\right),\left(S_{2}, 0.3\right),\left(S_{3}, 0.2\right)\right\}$ & -2.80 \\
$A_{2}$ & $\left\{\left(S_{3}, 0.22\right),\left(S_{4}, 0.6\right),\left(S_{5}, 0.18\right)\right\}$ & 0.93 \\
$A_{3}$ & $\left\{\left(S_{3}, 0.55\right),\left(S_{4}, 0.35\right),\left(S_{5}, 0.1\right)\right\}$ & 0.54 \\
\hline
\end{tabular}


Table 10 Distance of DM prospect values on alternatives following modification

\begin{tabular}{llll}
\hline & $d m^{1}$ & $d m^{2}$ & $d m^{3}$ \\
\hline$A_{1}$ & 1.10 & 0.85 & 1.45 \\
$A_{2}$ & 2.19 & 1.73 & 1.31 \\
$A_{3}$ & 0.37 & 0.34 & 0.24 \\
Total distance & 3.66 & 2.92 & 3.00 \\
\hline
\end{tabular}

$A_{3}$ is the best and is selected as the preferred designated medical institution. Moreover, if the outbreak continues and a hospital can no longer bear the medical pressure, hospitals $A_{2}$ and $A_{3}$ will be successively selected as the designated treatment hospitals.

\subsection{Analysis of Results}

In the above example, it is assumed that the values of $P\left(C_{o b j}\right)$ are all equal to 0.5 for $A_{1}, A_{2}$, and $A_{3}$ and that the DMs will take different values of $P\left(C_{o b j}\right)$ for different alternatives. Therefore, the following section analyzes the influence of consciousness flowing towards different evaluations on the result of the information, and provides several insights for determining the possible values of $P\left(C_{o b j}\right)$ in real decision making at two stages (the first stage concerns reference information acquisition and the second concerns modified information acquisition). We take $P\left(C_{o b j}\right) \in(0,1)$ with a step of 0.05 as the input, and through the data of $A_{1}, A_{2}$, and $A_{3}$, Figures 8, 9, and 10 can be obtained. Fig. (a) in Figs. 8, 9, and 10 indicates the reference information along the consciousness probability changes (the left vertical axis represents the distributions on the linguistic terms and the right one is the phase angle difference $\theta$ ). Furthermore, Fig. (b) in Figs. 8, 9, and 10 indicates the modified distributions on the linguistic terms of $A_{1}, A_{2}$, and $A_{3}$, respectively (the priority is still designed to be assigned for the distribution deviation of models (6) and (7) in the following analysis). The detailed analysis is as follows.

According to Fig. 8(a), each distribution exhibits an evident fluctuation during the interval $[0.35,0.5]$, and the interference becomes significantly negative from completely positive. in Fig. 8(b) shows the fluctuation during the interval $[0.05,0.2]$. As priority is assigned to the distribution deviation, we subsequently characterize the minimum distribution deviations on all linguistic terms between the reference and original information with different $P\left(C_{o b j}\right)$, which are listed in Table 11. It is demonstrated that $P\left(C_{o b j}\right)=0.45$ can decrease the deviation (from 0.4 to 0.26 ) effectively and obviously, and thereafter, the deviation remains at a relatively low level. The minimum deviation can be obtained when $P\left(C_{o b j}\right)=0.5$ with $\left\{\left(S_{1}, 0.47\right),\left(S_{2}, 0.23\right),\left(S_{3}, 0.3\right)\right\}$. If model (7) is adopted with the unknown $P\left(C_{o b j}\right)$, the optimized solution of this model is obtained as $\left\{\left(S_{1}, 0.5\right),\left(S_{2}, 0.2\right),\left(S_{3}, 0.3\right)\right\}$ with $P\left(C_{o b j}\right)=0.533, \theta=144^{\circ}$. Fig. 5 is a three-dimensional diagram that reveals the reference distributions along the phase angle difference changes when the probability of consciousness flowing towards objective information changes on $A_{1}$ for $d m^{1}$. Moreover, according to Fig. 8(b), the values of the distribution on different 

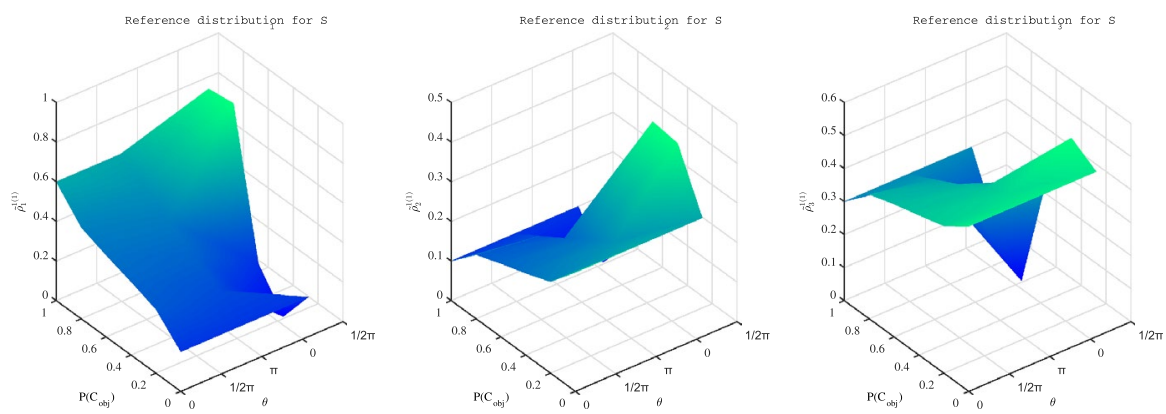

Fig. 5 Reference distributions along phase angle difference changes when probability of consciousness flowing towards objective information changes on $A_{1}$ for $d m^{1}$

linguistic terms are all stable in the interval [0.2, 0.9], which is equivalent to the original preference $d m^{1}$ being provided as $\left\{\left(S_{1}, 0.5\right),\left(S_{2}, 0.3\right),\left(S_{3}, 0.2\right)\right\}$. Under these circumstances, the distribution and expectation deviations between the original and modified prospect values are both equal to 0 . In summary, we can infer the following insight for determining $P\left(C_{o b j}\right)$ on $A_{1}$.

Insight on $A_{1}$ : Based on the principle that "the utility of the DMs is lower when they are further from the original opinion," $d m^{1}$ is more likely to prefer $P\left(C_{o b j}\right) \in[0.45,0.95]$ at the reference acquisition stage, and he/she would prefer $P\left(C_{o b j}\right) \in[0.2,0.9]$ at the modification acquisition stage. As there exists an overlap between $P\left(C_{o b j}\right) \in[0.45,0.95]$ and $P\left(C_{o b j}\right) \in[0.2,0.9]$, the common part $P\left(C_{o b j}\right) \in[0.45,0.9]$ is the most likely option in an actual decision environment, so as to guarantee the utilities of the two stages simultaneously.

According to Fig. 9(a), no fluctuation occurs for each distribution over the domain and no negative interference exists. Furthermore, the strength of the positivity continues to increase with the total positive interference from $P\left(C_{o b j}\right)=0.35$. Table 12 demonstrates that the distribution deviation maintains an upward trend on the entire domain, and thus, $d m^{1}$ will not prefer the larger $P\left(C_{o b j}\right)$. According to model (7), the optimized reference information is $\left\{\left(S_{4}, 0.6\right),\left(S_{5}, 0.15\right),\left(S_{6}, 0.25\right)\right\}$ with $P\left(C_{o b j}\right)=0$, which is consistent with the above findings. Under these situations, $\theta=90^{\circ}$, which means that the two classifications are independent, and the integration is equivalent to the Markov process. Fig. 6 is a three-dimensional diagram that reveals the reference distributions along the phase angle difference changes when the probability of consciousness flowing towards objective information changes on $A_{2}$ for $d m^{1}$. Furthermore, a smaller $P\left(C_{o b j}\right)$ indicates that the modified information is closer to the original preference, from the perspectives of both the distribution and the expectation deviations (as indicated in Table 13). Hence, we can conclude the findings regarding the value of $P\left(C_{o b j}\right)$ on $A_{2}$.

Insight on $A_{2}$ : Based on the principle that "the utility of the DMs is lower when they are further from the original opinion," a smaller value of $P\left(C_{o b j}\right)$ will be preferred by $d m^{1}$ with the reference and modification acquisition stages.

According to Fig. 10(a), the fluctuation generally appears around $P\left(C_{o b j}\right)=0.8$, with positive interference generated in most cases. The minimum distribution 

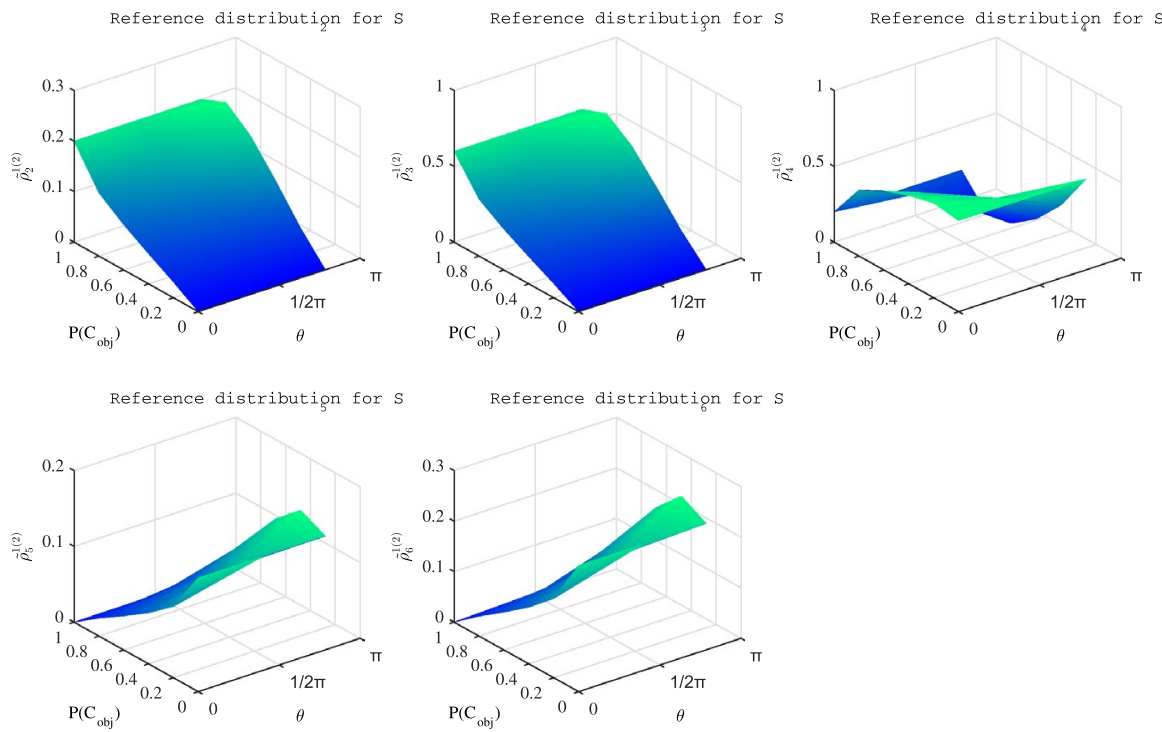

Fig. 6 Reference distributions along phase angle difference changes when probability of consciousness flowing towards objective information changes on $A_{2}$ for $d m^{1}$

deviations for all linguistic terms between the reference and original information with different $P\left(C_{o b j}\right)$ are listed in Table 14, which demonstrates that the distribution deviation decreases as $P\left(C_{o b j}\right)$ approaches 0.9. Thus, $\mathrm{dm}^{1}$ will prefer a larger $P\left(C_{o b j}\right)$ that is no more than 0.9 at the reference information acquisition stage. Using model (7), the optimized reference information is $\left\{\left(S_{3}, 0.5\right),\left(S_{4}, 0.4\right),\left(S_{5}, 0.1\right)\right\}$ with $P\left(C_{o b j}\right)=0.888$ and $\theta=180^{\circ}$, taking the minimum distribution deviation as 0.2 , which is consistent with the above findings in general. Fig. 7 is a three-dimensional diagram that reveals the reference distributions along the phase angle difference changes when the probability of consciousness flowing towards the objective information changes on $A_{3}$ for $d m^{1}$ (Figs. 8, 9). Moreover, in Fig. 10(b) is unstable overall. It can be observed that when $P\left(C_{o b j}\right)=0.8$, the modified information is closest to the original preference of $\mathrm{dm}^{1}$ as $\left\{\left(S_{3}, 0.4\right),\left(S_{4}, 0.5\right),\left(S_{5}, 0.1\right)\right\}$. In summary, we can infer the following insight for determining $P\left(C_{o b j}\right)$ on $A_{3}$.

Insight on $A_{3}$ : When the findings from the reference and modified information are not exactly the same, it is suggested that we should rely more on the modified information in this study, as this is the final preference that will be provided by the DMs. Therefore, the analysis demonstrates that $d m^{1}$ is more likely to take $P\left(C_{o b j}\right)=0.8$ on $A_{3}$.

The above analysis focuses on the most likely attitudes towards different reference information paths for a certain alternative. Under the assumption of the unified $P\left(C_{o b j}\right)$ for all alternatives, we should pay attention to the overall performance. Table 15 presents the total expectation and distribution deviations between the modified and original information for different $P\left(C_{o b j}\right)$ for all alternatives, which are 

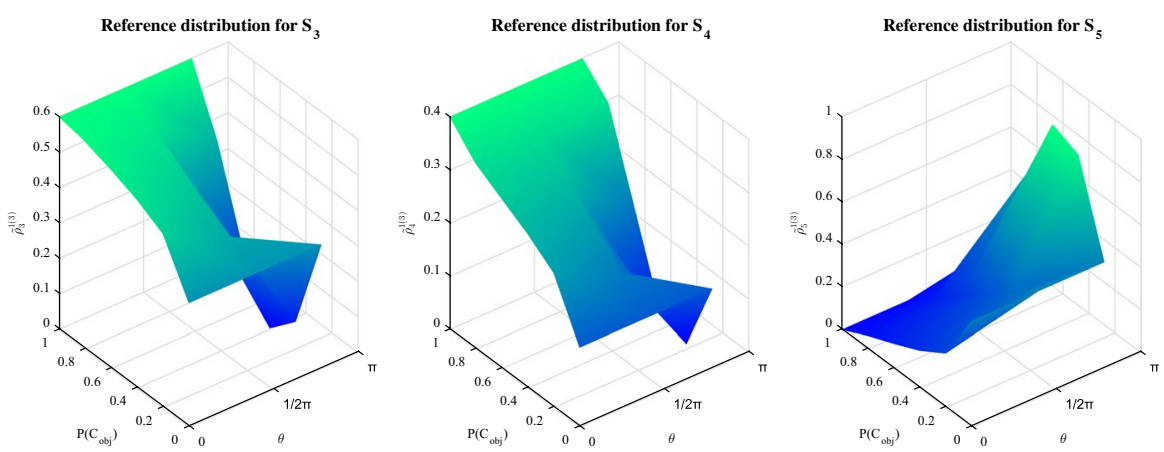

Fig. 7 Reference distributions along phase angle difference changes when probability of consciousness flowing towards objective information changes on $A_{3}$ for $d^{1}$

Table 11 Distribution deviation between reference and original information for different $P\left(C_{o b j}\right)$ on $A_{1}$

\begin{tabular}{llllllllllll}
\hline$P\left(C_{\text {obj }}\right)$ & 0.05 & 0.1 & 0.15 & 0.2 & 0.25 & 0.3 & 0.35 & 0.4 & 0.45 & 0.5 & 0.55 \\
\hline Distribution deviation & 0.54 & 0.52 & 0.51 & 0.49 & 0.48 & 0.47 & 0.46 & 0.40 & 0.26 & 0.20 & 0.22 \\
\hline$P\left(C_{o b j}\right)$ & 0.6 & 0.65 & 0.7 & 0.75 & 0.8 & 0.85 & 0.9 & 0.95 & & & \\
\hline Distribution deviation & 0.26 & 0.28 & 0.29 & 0.30 & 0.31 & 0.31 & 0.31 & 0.33 & & & \\
\hline
\end{tabular}

(a) Reference information

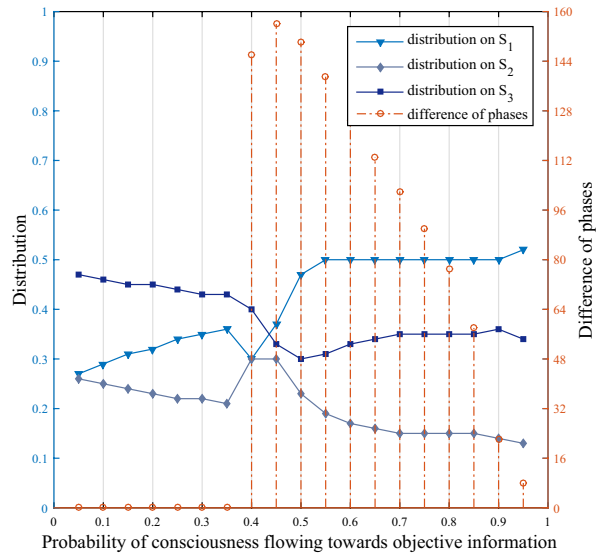

(b) Modified information

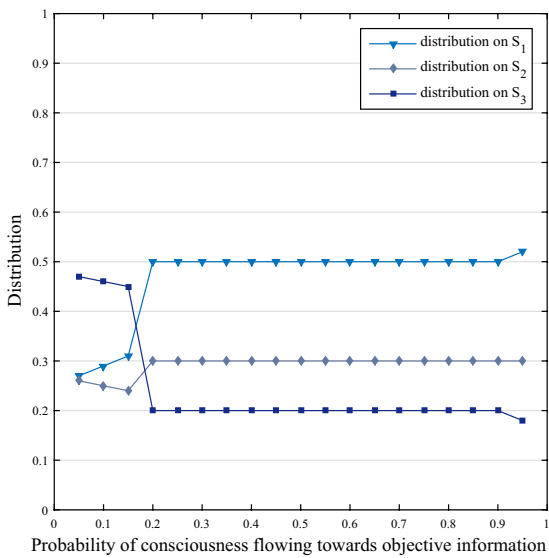

Fig. 8 Relevant information of $A_{1}$ along consciousness probability changes

visually represented in Fig. 11. Evidently, the total utilities of the DMs reach the highest level when $P\left(C_{o b j}\right)=0.2$.

According to the above, the final utility of the DM depends on the distance of the modified information from the original preference, whereas the moderator, whose aim is to promote group consensus, hopes that the prospect values of all DMs on 
(a) Reference information

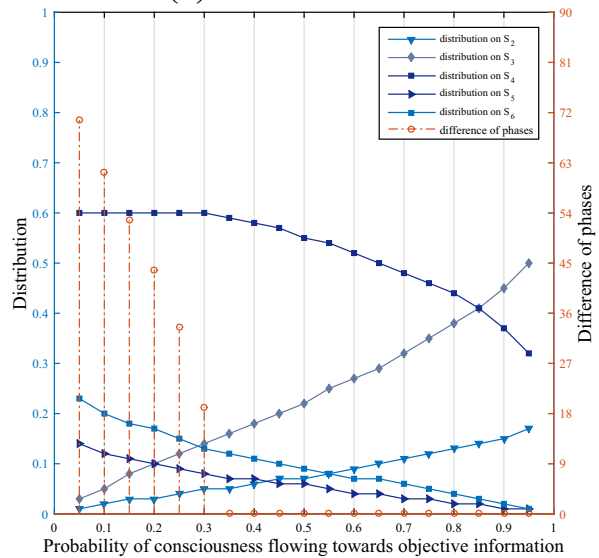

(b) Modified information

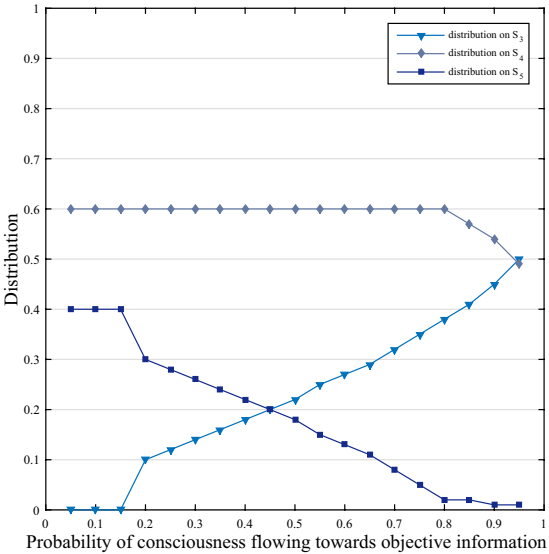

Fig. 9 Relevant information of $A_{2}$ along consciousness probability changes

Table 12 Distribution deviation between reference and original information for different $P\left(C_{o b j}\right)$ on $A_{2}$

\begin{tabular}{llllllllllll}
\hline$P\left(C_{\text {obj }}\right)$ & 0.05 & 0.1 & 0.15 & 0.2 & 0.25 & 0.3 & 0.35 & 0.4 & 0.45 & 0.5 & 0.55 \\
\hline Distribution deviation & 0.53 & 0.55 & 0.58 & 0.60 & 0.62 & 0.64 & 0.67 & 0.70 & 0.74 & 0.78 & 0.82 \\
\hline$P\left(C_{\text {obj }}\right)$ & 0.6 & 0.65 & 0.7 & 0.75 & 0.8 & 0.85 & 0.9 & 0.95 & & & \\
\hline Distribution deviation & 0.87 & 0.91 & 0.96 & 1.02 & 1.08 & 1.15 & 1.23 & 1.34 & & & \\
\hline
\end{tabular}

Table 13 Deviations between modified and original information for different $P\left(C_{o b j}\right)$ on $A_{2}$

\begin{tabular}{llllllllllll}
\hline$P\left(C_{\text {obj }}\right)$ & 0.05 & 0.1 & 0.15 & 0.2 & 0.25 & 0.3 & 0.35 & 0.4 & 0.45 & 0.5 & 0.55 \\
\hline Expectation deviation & 0.00 & 0.00 & 0.00 & 0.20 & 0.24 & 0.28 & 0.32 & 0.36 & 0.40 & 0.44 & 0.50 \\
Distribution deviation & 0.00 & 0.00 & 0.00 & 0.20 & 0.24 & 0.28 & 0.32 & 0.36 & 0.40 & 0.44 & 0.50 \\
\hline$P\left(C_{\text {obj }}\right)$ & 0.6 & 0.65 & 0.7 & 0.75 & 0.8 & 0.85 & 0.9 & 0.95 & & & \\
\hline Expectation deviation & 0.54 & 0.58 & 0.64 & 0.70 & 0.76 & 0.82 & 0.90 & 1.00 & & & \\
Distribution deviation & 0.54 & 0.58 & 0.64 & 0.70 & 0.76 & 0.79 & 0.84 & 0.89 & & & \\
\hline
\end{tabular}

Table 14 Distribution deviation between reference and original information for different $P\left(C_{o b j}\right)$ on $A_{3}$

\begin{tabular}{llllllllllll}
\hline$P\left(C_{\text {obj }}\right)$ & 0.05 & 0.1 & 0.15 & 0.2 & 0.25 & 0.3 & 0.35 & 0.4 & 0.45 & 0.5 & 0.55 \\
\hline Distribution deviation & 0.59 & 0.55 & 0.52 & 0.49 & 0.47 & 0.45 & 0.44 & 0.42 & 0.40 & 0.39 & 0.37 \\
\hline$P\left(C_{o b j}\right)$ & 0.6 & 0.65 & 0.7 & 0.75 & 0.8 & 0.85 & 0.9 & 0.95 & & & \\
\hline Distribution deviation & 0.36 & 0.35 & 0.33 & 0.32 & 0.30 & 0.25 & 0.24 & 0.33 & & & \\
\hline
\end{tabular}


(a) Reference information

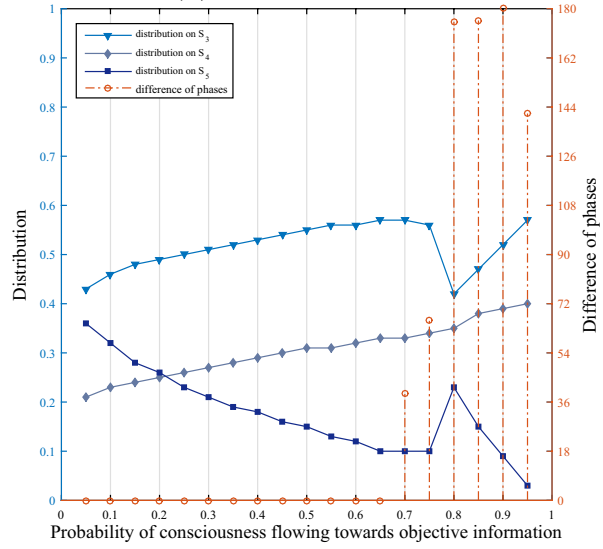

(b) Modified information

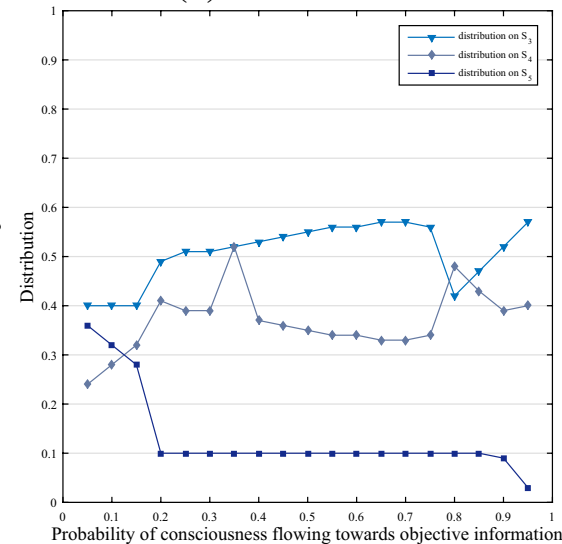

Fig. 10 Relevant information of $A_{3}$ along consciousness probability changes

Table 15 Total deviations between modified and original information for different $P\left(C_{o b j}\right)$ on all alternatives

\begin{tabular}{llllllllllll}
\hline$P\left(C_{o b j}\right)$ & 0.05 & 0.1 & 0.15 & 0.2 & 0.25 & 0.3 & 0.35 & 0.4 & 0.45 & 0.5 & 0.55 \\
\hline Expectation deviation & 0.76 & 0.69 & 0.62 & 0.29 & 0.35 & 0.39 & 0.76 & 0.49 & 0.54 & 0.59 & 0.66 \\
Distribution deviation & 1.06 & 0.96 & 0.86 & 0.38 & 0.46 & 0.50 & 0.46 & 0.62 & 0.68 & 0.74 & 0.82 \\
\hline$P\left(C_{o b j}\right)$ & 0.6 & 0.65 & 0.7 & 0.75 & 0.8 & 0.85 & 0.9 & 0.95 & & & \\
\hline Expectation deviation & 0.70 & 0.75 & 0.81 & 0.86 & 0.78 & 0.86 & 0.97 & 1.17 & & & \\
Distribution deviation & 0.86 & 0.92 & 0.98 & 1.02 & 0.80 & 0.96 & 1.14 & 1.38 & & & \\
\hline
\end{tabular}

Table 16 Total deviations of prospect values between $d m^{1}$ and other DMs on all alternatives with dynamic reference point

\begin{tabular}{llllllllllll}
\hline$P\left(C_{o b j}\right)$ & 0.05 & 0.1 & 0.15 & 0.2 & 0.25 & 0.3 & 0.35 & 0.4 & 0.45 & 0.5 & 0.55 \\
\hline Prospect values deviation & 3.86 & 4.06 & 4.26 & 4.23 & 4.11 & 4.04 & 3.95 & 3.85 & 3.76 & 3.66 & 3.53 \\
\hline$P\left(C_{o b j}\right)$ & 0.6 & 0.65 & 0.7 & 0.75 & 0.8 & 0.85 & 0.9 & 0.95 & & & \\
\hline Prospect values deviation & 3.46 & 3.36 & 3.25 & 3.16 & 3.33 & 3.17 & 2.96 & 2.48 & & & \\
\hline
\end{tabular}

each alternative are as close as possible. The relevant data are displayed in Table 16 and Fig. 11. According to the orange broken line, which reflects the deviation in the prospect value in Fig. 11, the peak appears at $P\left(C_{o b j}\right)=0.15$, which is the point that the moderator is most reluctant towards. Combined with the above analysis, the conflict between the DMs and moderator is generated. The value of the most likely $P\left(C_{o b j}\right)$ following the game confrontation between the moderator and DMs is worth studying in the future. The $P\left(C_{o b j}\right)$ with the minimum values of the prospect 


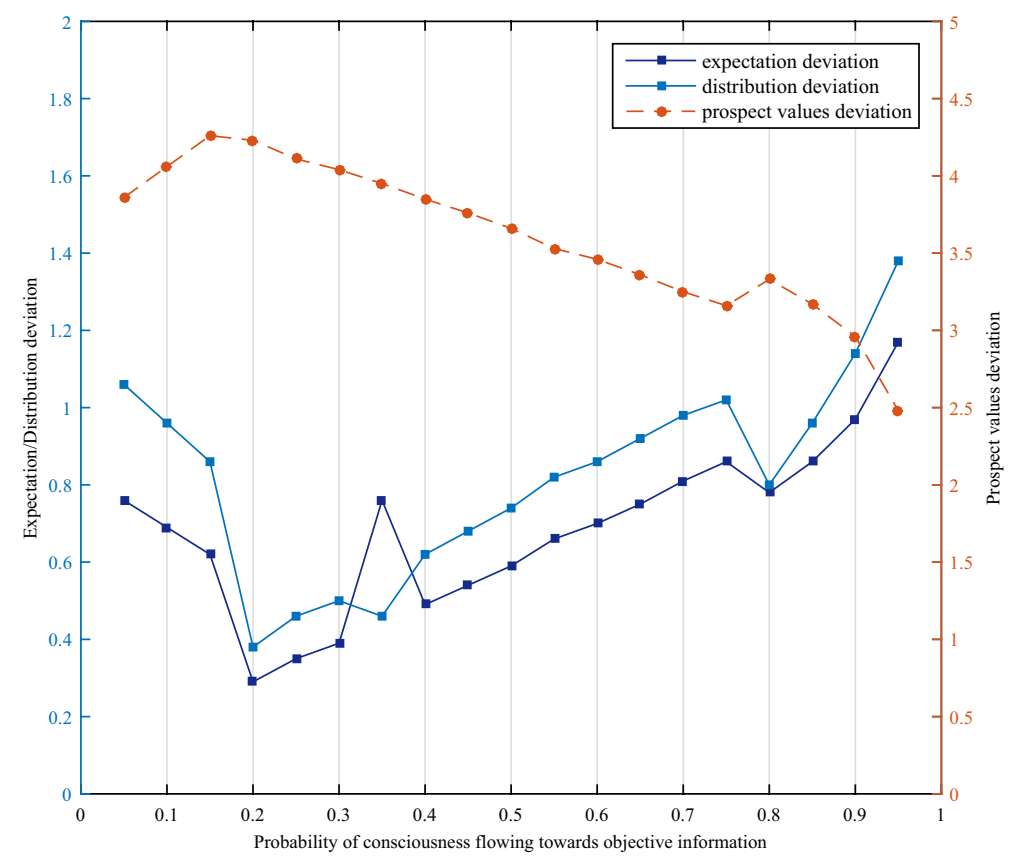

Fig. 11 Relevant deviations of modified preference along consciousness probability changes

deviation and expectation/distribution deviations appearing simultaneously is the most satisfied attitude towards the objective information in the process of refereeing information for the DMs and moderators. Moreover, the total deviations of the prospect values between the $d m^{1}$ and other DMs for all alternatives in Table 16 are all less than the original deviation of 6.36 in Table 5, which proves the validity of the proposed consensus model.

In summary, the simulation can aid in estimating the most likely value of $P\left(C_{o b j}\right)$ based on certain rules for the DMs and moderator. In the above analysis, the priority is assigned for the distribution deviation and the situation of the priority arrangement for the expectation distribution can be similarly explored.

\subsection{Comparative Analysis}

To elucidate the advantages of the proposed CRP, we conducted a comparative analysis on multiple objectives in models (6) and (7) regarding the value of the interference term in the QPT. Furthermore, we analyzed the efficiency of the proposed consensus model compared to the existing consensus method based on the dynamic reference point. We present the comparative analysis with $\mathrm{Li}$ et al. (2021) in terms of the linguistic distribution context, and analyze the linkages between model (6), model (13), and the existing works (Zhang et al. 2018; Wu et al. 2018, 2020). 


\subsubsection{Comparative Analysis Regarding Multiple Objectives in Models (6) and (7)}

The function of model (6) is to make the rough information accurate. Considering the rough subjective information on $A_{3}:\left\{\left(S_{3}, 0.35\right),\left(S_{4}, 0.15\right),\left(S_{5}, 0.3\right),\left(S_{6}\right.\right.$, $0.3)\}$ as an example to illustrate the advantage and necessity of setting multiple objectives, first, the situation with the priority assigned for the distribution objective function is analyzed. It is known that the optimized assessment obtained by model (6) is $\left\{\left(S_{3}, 0.35\right),\left(S_{4}, 0.15\right),\left(S_{5}, 0.5\right)\right\}$ with a distribution deviation of 0.5 and an expectation deviation of 0.8 . When only the distribution objective function is considered as in Zhang et al. (2018), $\left\{\left(S_{3}, 0.35\right),\left(S_{4}, 0.35\right),\left(S_{5}, 0.3\right)\right\}$ can be obtained with a distribution deviation of 0.5 and an expectation deviation of 1. Obviously, the expectation deviation is narrowed by multiple objectives with the same distribution deviation. Second, the situation with the priority assigned to the expectation objective function is analyzed: $\left\{\left(S_{4}, 0.35\right),\left(S_{5}, 0.35\right),\left(S_{6}, 0.3\right)\right\}$ can be obtained through multiple objectives with a distribution deviation of 0.6 and an expectation deviation of 0 . When only the expectation objective function is considered as in Tan et al. (2021), $\left\{\left(S_{4}, 0.52\right),\left(S_{5}, 0.01\right),\left(S_{6}, 0.47\right)\right\}$ can be obtained with a distribution deviation of 1.18 and an expectation deviation of 0 . Obviously, the distribution deviation is narrowed by multiple objectives with the same expectation deviation. In conclusion, regardless of which objective function is assigned with priority, the optimal solution that minimizes the distribution deviation and the expected deviation can be determined through multiple objectives among feasible solutions. Therefore, the construction of multiple objectives in model (6) is effective. The similar analysis for model (7) is omitted here.

\subsubsection{Comparative Analysis Regarding Value of Interference Term in QPT}

Considering that there is not yet an authoritative method for determining the value of $\theta$ (He et al. 2018), our proposal constructs a multi-objective programming model in the form of model (7) on the basis of individual utility in CRP to obtain the solution. If the similarity heuristic method proposed in Catarina and Andreas (2016) is adopted, we can obtain the difference of the phases For $A_{1}, \theta=67.59^{\circ}$, which means that the interference is positive to a certain extent. For $A_{2}, \theta=107.76^{\circ}$, which means that the interference is relatively mildly negative. For $A_{3}, \theta=75.64^{\circ}$, which means that the interference is positive to a certain extent, being closer to independence compared to $\theta=67.59^{\circ}$ on $A_{1}$. There is an evident difference compared to the outcomes presented in Sect. 5.1.3. The modification information range can be obtained as $\rho_{1}^{1(1)} \in[0.39,0.5], \rho_{2}^{1(1)} \in[0.2,0.3]$, and $\rho_{3}^{1(1)} \in[0.2,0.4]$ with all the other distributions equal to zero. Obviously, the modification range is extended compared to that in Table 8 , and the utility of the individual will be negatively affected. Therefore, the adoption of the goal programming model can aid in obtaining an optimized quantum integration solution based on the maximum satisfaction level of the individual. 


\subsubsection{Efficiency of Proposed Model Compared to Existing Consensus Methods Based on Dynamic Reference Point}

In the consensus process with the application of PT, the reference point is supposed to be re-established following the preference modification, rather than dynamic during the adjustment (Wang et al. 2015; Gao et al. 2017), which is considered in this study. The calculated numerical example in this study reflects this view in detail. The modified information on $A_{2}$ takes different minimum linguistic terms under different situations, resulting in different reference points, and the prospect value is formed based on the dynamic reference point. It can be determined that the reference point is $S_{4}$ with $P\left(C_{o b j}\right) \in[0.05,0.15]$ and is $S_{3}$ with $P\left(C_{o b j}\right) \in[0.2,0.95]$ based on the "MaxMin" rule for $A_{2}$ from in Fig. 9(b), which indicates the dynamic characteristics of the reference point.

If we adopt the proposal in Wang et al. (2015) and Gao et al. (2017), that is, the reference point is stable during modification for each iteration, the dynamic characteristic of the reference point is reflected in the fact that it will change when entering the next round of modification. Thus, Table 17 can be provided, which demonstrates the total deviation of the prospect values for each DM on all alternatives (i.e. $d^{k}$ ) with different values of $P\left(C_{o b j}\right)$ after the first modification based on the reference point $\left(S_{4}\right)$. Table 18 provides the relevant information with the dynamic reference point. It is obvious that there exists $d^{k}>\phi(4.5)$ in Table 17; thus, the CRP needs to be conducted again. However, with the dynamic reference point during adjustment, Table 18 indicates that only one modification is sufficient, as all $d^{k}<\phi(4.5)$ and the ending condition of the CRP is reached. The numerical results indicate that our method is more efficient with fewer rounds of modification compared to existing methods.

\subsubsection{Comparative Analysis with Li et al. (2021) Regarding Linguistic Distribution Context}

This study investigates the CRP in the linguistic distribution context based on the relevant behavioral decision theories through series optimization models, whereas Li

Table 17 Total deviation of prospect values for each DM on all alternatives with initial reference point $S_{4}$ following first modification

\begin{tabular}{llllllllllll}
\hline$P\left(C_{o b j}\right)$ & 0.05 & 0.1 & 0.15 & 0.2 & 0.25 & 0.3 & 0.35 & 0.4 & 0.45 & 0.5 & 0.55 \\
\hline$d m^{1}$ & 3.67 & 3.74 & 3.74 & 3.69 & 3.81 & 3.93 & 4.01 & 3.92 & 4.51 & 5.04 & 5.15 \\
$d m^{2}$ & 3.43 & 3.46 & 3.46 & 3.43 & 3.5 & 3.56 & 3.6 & 3.55 & 3.85 & 4.11 & 4.17 \\
$d m^{3}$ & 2.5 & 2.53 & 2.53 & 2.51 & 2.57 & 2.63 & 2.7 & 2.62 & 2.92 & 3.18 & 3.24 \\
\hline$P\left(C_{o b j}\right)$ & 0.6 & 0.65 & 0.7 & 0.75 & 0.8 & 0.85 & 0.9 & 0.95 & & & \\
\hline$d m^{1}$ & 5.09 & 5.09 & 5.05 & 5.05 & 4.79 & 4.95 & 5.01 & 5.09 & & \\
$d m^{2}$ & 4.14 & 4.14 & 4.12 & 4.12 & 3.99 & 4.07 & 4.1 & 4.14 & & \\
$d m^{3}$ & 3.21 & 3.21 & 3.19 & 3.19 & 3.06 & 3.14 & 3.17 & 3.21 & & & \\
\hline
\end{tabular}


Table 18 Total deviation of prospect values for each DM on all alternatives with dynamic reference point following first modification

\begin{tabular}{llllllllllll}
\hline$P\left(C_{\text {obj }}\right)$ & 0.05 & 0.1 & 0.15 & 0.2 & 0.25 & 0.3 & 0.35 & 0.4 & 0.45 & 0.5 & 0.55 \\
\hline$d m^{1}$ & 3.86 & 4.06 & 4.26 & 4.23 & 4.13 & 4.04 & 3.95 & 3.85 & 3.76 & 3.66 & 3.53 \\
$d m^{2}$ & 3.52 & 3.62 & 3.72 & 3.2 & 3.16 & 3.11 & 3.06 & 3.01 & 2.97 & 2.92 & 2.85 \\
$d m^{3}$ & 2.6 & 2.69 & 2.79 & 3.28 & 3.23 & 3.19 & 3.14 & 3.09 & 3.04 & 3 & 2.93 \\
\hline$P\left(C_{\text {obj }}\right)$ & 0.6 & 0.65 & 0.7 & 0.75 & 0.8 & 0.85 & 0.9 & 0.95 & & & \\
\hline$d m^{1}$ & 3.46 & 3.36 & 3.25 & 3.16 & 3.33 & 3.17 & 2.96 & 2.53 & & & \\
$d m^{2}$ & 2.82 & 2.77 & 2.72 & 2.67 & 2.75 & 2.67 & 2.57 & 2.36 & & & \\
$d m^{3}$ & 2.9 & 2.85 & 2.79 & 2.75 & 2.83 & 2.75 & 2.64 & 2.43 & & & \\
\hline
\end{tabular}

et al. (2021) focused on the estimation of the missing distribution with personalized individual semantics (PISs) in linguistic distribution GDM based on the consistency condition through collective/individual optimization models. Several insights can be obtained for future studies by comparing the two references. The comparison is presented as follows and is summarized in Table 19.

First, the preference relations in $\mathrm{Li}$ et al. (2021) are pairwise comparison preference relations, which are equipped with the consistency condition to measure the rationality and logic of the relations matrix. As this study focuses on multi-attribute preference relations, consistency is not involved in our decision problem. The proposed CRP with behavioral theories can be further researched using pairwise comparison preference relations. This is owing to the consistency condition in Li et al. (2021) that can obtain the missing judgments based on the logic of preference relations, whereas the elements in preference relations are complete in our paper. When ignorance elements exist in multi-attribute preference relations, a possible method for their estimation may be to integrate all other DM preferences on the missing position using QPT. The PISs regarding the words adopted in Li et al. (2021) is flexible and personalized compared to the computation of the linguistic term in this study, which is more in accordance with the actual decision-making environment. Optimization models are constructed in both papers to obtain the preference information, with the best consistency as the objective

Table 19 Comparison between Li et al. (2021) and this study

\begin{tabular}{|c|c|c|}
\hline & Li et al. (2021) & This paper \\
\hline Preference relations & Pairwise comparison & Multi-attribute preference relations \\
\hline Elements in preference relations & Incomplete & Complete \\
\hline Linguistic numerical scale & Personalized & Fixed and traditional \\
\hline Aim & $\begin{array}{l}\text { computation of ignorance ele- } \\
\text { ments and PISs }\end{array}$ & Consensus reaching \\
\hline Rule used & Consistency condition & Behavioral theories \\
\hline Environment & Linguistic distribution context & Linguistic distribution context \\
\hline Methodology & Optimization model & Optimization model \\
\hline
\end{tabular}


in Li et al. (2021), and the minimum modification and best consensus as the objectives in this paper.

Moreover, it can be observed that the complete preference information acquired from the optimization models in $\mathrm{Li}$ et al. (2021) does not focus on the information accuracy (Zhang et al. 2018; Wu et al. 2018, 2020). For example, the probabilistic information in Table 5 in Li et al. (2021) for $\bar{p}_{12, t}^{1}, \bar{p}_{13, t}^{1}, \bar{p}_{14, t}^{1}$ takes a non-zero value for each linguistic term, and for $\bar{p}_{23, t}^{1}, \bar{p}_{24, t}^{1}, \bar{p}_{34, t}^{1}$, the succession performance is not sufficient good, which is inaccurate as mentioned in Zhang et al. (2018) and Wu et al. (2018, 2020). Also, The preference attitude introduced in this study for all six preference information sets is opposite in one linguistic distribution assessment. It is proposed that the quality of the preference information should be guaranteed in this study.

\subsubsection{Linkages Between Model (6), Model (13), and Existing Works (Zhang et al. 2018; Wu et al. 2018, 2020)}

As described above, models (6) and (13) in this study are established based on Zhang et al. (2018) and Wu et al. (2018, 2020), with the distribution linguistic term assessment. Optimization models are constructed in all three works to prompt the CRP, and the common operation of the existing models is that the accurate constraints (i.e. constraints (1) in this study) are developed to improve the quality of the collective (aggregated) preference information, which is adopted in models (6) and (13) to guarantee the accuracy of the preference information. Furthermore, this study introduces the concept of "attitude consistency" on the basis of the commonly used constraints, thereby ensuring the accuracy of the preference information from the perspective of individual attitudes so as to cause the consensus model to exhibit universality when the number of involved linguistic terms is more than 2 (i.e. $N \geq 3$ ).

In terms of objective functions, Wu et al. (2018) aimed at maximizing the support degree of the group opinion, whereas Zhang et al. (2018) and Wu et al. (2020) both focused on the minimum preference loss to support the CRP. Similarly, model (6) aims for the collective preference to be as close as possible to the individual assessment. We introduce the excepted value together with the distribution with which existing models are concerned to construct a multi-objective function. This advantage is verified in Sect. 5.3.1. Moreover, model (13) is aimed at the minimum distance between the individual prospect values on all alternatives with the designed mechanism of the dynamic reference point that is involved in the constraints. To recapitulate, the proposed models (6) and (13) develop the accuracy conditions that the distribution linguistic assessment should obey, construct the multi-objective function to obtain better preference information, and introduce objective functions from different perspectives based on existing works.

\section{Conclusions}

Our study suggests the possibility of quantum consciousness during the integration of different information paths, and establishes a multi-objective programming model to determine the interference term conforming to the psychological utility of the 
DMs, thereby demonstrating that the proposed model is more reliable than existing methods from a behavioral perspective. Furthermore, this study introduces identification based on the prospect value and dynamic reference point in the modification process, which considers the dynamic characteristics of the behavioral decisionmaking process and eliminates the destabilization of various complex factors on the decision-making accuracy.

Overall, this study offers a new strategy to consider the complexity of the linguistic distribution decision-making environment and individual non-rationality, as well as the conflict, uncertainty, and fuzziness that are reflected in the brain. However, there are also limitations to this study. First, the reference information is simulated based on the assumptive quantum mechanism and mathematical model; however, the consciousness of human behavior is elusive. Whether other uncertain factors need to be analyzed is a topic worth exploring. Second, we only focus on the dynamic property when the DM makes modifications; however, the expectations and bottom lines of DMs will change with the continual development of decisionmaking events. The issue of dynamic behavioral characteristics at different stages is an interesting research topic.

Acknowledgements This work has been supported by the National Natural Science Foundation of China under Grant 72071106, Grant 72074001, and Grant 71601002, Grant 72001111; the Postgraduate Research \& Practice Innovation Program of Jiangsu Province under Grant KYCX21_0238; Natural Science Foundation of Jiangsu Province (BK20210293).

\section{Declarations}

Conflict of interest The authors declare that they have no known competing financial interests or personal relationships that could have appeared to influence the work reported in this paper.

\section{References}

Aerts D, Aerts S (1995) Applications of quantum statistics in psychological studies of decision processes. Found Sci 1(1):85-97

Aerts D, Sozzo S (2012) Quantum structure in economics: the ellsberg paradox. Am Inst Phys Conf Proc 1424(1):487-494

Baillon A, Bleichrodt H, Spinu V (2020) Searching for the reference point. Manag Sci 66(1):93-112

Bezdek JC, Spillman B, Spillman R (1978) A fuzzy relation space for group decision theory. Fuzzy Sets Syst 1(4):255-268

Busemeyer JR, Wang Z (2018) Data fusion using hilbert space multi-dimensional models. Theor Comput Sci 752:41-55

Busemeyer JR, Wang Z, Lambert-Mogiliansky A (2009) Empirical comparison of markov and quantum models of decision-making. J Math Psychol 53(5):423-433

Busemeyer JR, Wang Z, Shiffrin RM (2015) Bayesian model comparison favors quantum over standard decision theory account of dynamic inconsistency. Decision 2(1):1-12

Catarina M, Andreas W (2016) Quantum-like bayesian networks for modeling decision making. Front Psychol 7:11

Ding RX, Wang XQ, Shang K, Herrera F (2019) Social network analysis-based conflict relationship investigation and conflict degree-based consensus reaching process for large scale decision making using sparse representation. Inf Fusion 50:251-272

Ding XF, Liu HC, Shi H (2019) A dynamic approach for emergency decision making based on prospect theory with interval-valued pythagorean fuzzy linguistic variables. Comput Ind Eng 131:57-65 
Dolan P, Robinson A (2001) The measurement of preferences over the distribution of benefits: the importance of the reference point. Eur Econ Rev 45(9):1697-1709

Dong YC, Zhang HJ, Herrera-Viedma E (2016) Integrating experts' weights generated dynamically into the consensus reaching process and its applications in managing non-cooperative behaviors. Decis Support Syst 84:1-15

Fan ZP, Ma J, Zhang Q (2002) An approach to multiple attribute decision making based on fuzzy preference information on alternatives. Fuzzy Sets Syst 131:101-106

Gao J, Xu ZS, Liao HC (2017) A dynamic reference point method for emergency response under hesitant probabilistic fuzzy environment. Int J Fuzzy Syst 19(5):1261-1278

Gong ZW, Zhang HH, Forrest J, Li LS, Xu XX (2015) Two consensus models based on the minimum cost and maximum return regarding either all individuals or one individual. Eur J Operation Res 240(1):183-192

He ZC, Chan FTS, Jiang W (2018) A quantum framework for modelling subjectivity in multi-attribute group decision making. Comput Ind Eng 124:560-572

Herrera F, Herrera-Viedma E (2000) Linguistic decision analysis: steps for solving decision problems under linguistic information. Fuzzy Sets Syst 115(1):67-82

Jing X, Wang XL, Zhang HJ (2020) Managing classification-based consensus in social network group decision making: an optimization-based approach with minimum information loss. Inf Fusion 63:74-87

Kamis NH, Chiclana F, Levesley J (2017) Preference similarity network structural equivalence clustering based consensus group decision making model. Appl Soft Comput 67:706-720

Khrennikov A (1999) Classical and quantum mechanics on information spaces with applications to cognitive, psychological, social, and anomalous phenomena. Found Phys 29(7):1065-1098

Labella Á, Liu YY, Rodríguez RM, Martínez L (2018) Analyzing the performance of classical consensus models in large scale group decision making: a comparative study. Appl Soft Comput 67:677-690

Labella Á, Rodríguez RM, Alzahrani AA, Martínez L (2020) A consensus model for extended comparative linguistic expressions with symbolic translation. Mathematics 8(12):2198

Li CC, Gao Y, Dong YC (2021) Managing ignorance elements and personalized individual semantics under incomplete linguistic distribution context in group decision making. Group Decis Negotiat 30(1):97-118

Li CC, Rodríguez RM, Martínez L, Dong YC, Herrera F (2019) Consensus building with individual consistency control in group decision making. IEEE Trans Fuzzy Syst 27(2):319-332

Liu BS, Zhou Q, Ding RX, Palomares I, Herrera F (2019) Large-scale group decision making model based on social network analysis: trust relationship-based conflict detection and elimination. Eur J Operation Res 275(2):737-754

Liu Y, Zhou T, Forrest YL (2020) A multivariate minimum cost consensus model for negotiations of holdout demolition. Group Decis Negotiat 29(5):871-899

Neumann JV, Morgenstern O (1944) Theory of games and economic behavior. Princeton University Press, Princeton

Rodríguez RM, Labella Á, Tré GD, Martínez L (2018) A large scale consensus reaching process managing group hesitation. Knowl-Based Syst 159:86-97

Song W, Zhu JJ (2019) Three-reference-point decision-making method with incomplete weight information considering independent and interactive characteristics. Inf Sci 503:148-168

Tan X, Zhu JJ, Cabrerizo FJ, Herrera-Viedma E (2021) A cyclic dynamic trust-based consensus model for large-scale group decision making with probabilistic linguistic information. Appl Soft Comput 100(2): 106937

Tversky A, Kahneman D (1979) Prospect theory: an analysis of decision under risk. Econometrica 47(2):263-291

Tversky A, Kahneman D (1992) Advances in prospect theory: cumulative representation of uncertainty. J Risk Uncertain 5(4):297-323

Wang L, Zhang ZX, Wang YM (2015) A prospect theory-based interval dynamic reference point method for emergency decision making. Expert Syst Appl 42:9379-9388

Wang Z, Busemeyer JR (2016) Interference effects of categorization on decision making. Cognition 150:133-149

Wang Z, Solloway T, Shiffrin RM, Busemeyer JR (2014) Context effects produced by question orders reveal quantum nature of human judgments. Proc Natl Acad Sci 111(26):9431-9436

Wu T, Liu XW, Qin JD, Herrera F (2019) Consensus evolution networks: a consensus reaching tool for managing consensus thresholds in group decision making. Inf Fusion 52:375-388 
Wu YZ, Dong YC, Qin JD, Pedrycz W (2020) Flexible linguistic expressions and consensus reaching with accurate constraints in group decision-making. IEEE Trans Cybernet 50(6):2488-2501

Wu YZ, Li CC, Chen X, Dong YC (2018) Group decision making based on linguistic distributions and hesitant assessments: Maximizing the support degree with an accuracy constraint. Inf Fusion 2018(41):151-160

Wu ZB, Xu JP (2018) A consensus model for large-scale group decision making with hesitant fuzzy information and changeable clusters. Inf Fusion 41:217-231

Xu WJ, Chen X, Dong YC, Chiclana F (2021) Impact of decision rules and non-cooperative behaviors on minimum consensus cost in group decision making. Group Decis Negotiat 30(6):1239-1260

Xu WJ, Huang SY, Li J (2018) A novel consensus reaching framework for heterogeneous group decision making based on cumulative prospect theory. Comput Ind Eng 128:325-335

Yukalov VI, Sornette D (2012) Quantum decision theory as quantum theory of measurement. Phys Lett A 372(46):6867-6871

Zadeh LA (1975) The concept of a linguistic variable and its application to approximate reasoning-I. Inf Sci 8(3):199-249

Zhang BW, Dong YC, Herrera-Viedma E (2019) Group decision making with heterogeneous preference structures: an automatic mechanism to support consensus reaching. Group Decis Negotiat 28:585-617

Zhang BW, Liang HM, Gao Y, Zhang GQ (2018) The optimization-based aggregation and consensus with minimum-cost in group decision making under incomplete linguistic distribution context. Knowl-Based Syst 162:92-102

Zhang GQ, Dong YC, Xu YF (2014) Consistency and consensus measures for linguistic preference relations based on distribution assessments. Inf Fusion 17:46-55

Zhang ST, Zhu JJ, Liu XD, Chen Y (2016) Regret theory-based group decision-making with multidimensional preference and incomplete weight information. Inf Fusion 31:1-13

Zhang YX, Xu ZS, Liao HC (2020) A consensus process for group decision making with probabilistic linguistic preference relations. Inf Sci 533:150-168

Zhou XY, Wang LQ, Liao HC, Wang SY, Lev B, Fujita H (2019) A prospect theory-based group decision approach considering consensus for portfolio selection with hesitant fuzzy information. KnowlBased Syst 168:28-38

Zhu JJ, Ma ZZ, Wang HH, Chen Y (2017) Risk decision-making method using interval numbers and its application based on the prospect value with multiple reference points. Inf Sci 385:415-437

Publisher's Note Springer Nature remains neutral with regard to jurisdictional claims in published maps and institutional affiliations.

\title{
Authors and Affiliations
}

\author{
Xiao $\operatorname{Tan}^{1} \cdot$ Jianjun $\mathrm{Zhu}^{1}{ }^{\text {(D) }} \cdot$ Tong $\mathrm{Wu}^{1}$ \\ Xiao Tan \\ ctanxiao@163.com \\ Tong Wu \\ tongwu@nuaa.edu.cn \\ 1 College of Economics and Management, Nanjing University of Aeronautics and Astronautics, \\ Nanjing, Jiangsu, China
}

